\title{
van Vleck determinants: traversable wormhole spacetimes
}

\author{
Matt Visser日 \\ Physics Department, Washington University, St. Louis, Missouri 63130-4899
}

(5 October 1993)

\begin{abstract}
Prompted by Hawking's chronology protection conjecture, and by various other questions regarding the putative existence, stability, and chronological properties of traversable wormholes, a number of authors have presented calculations of the renormalized stress-energy tensor in wormhole spacetimes. In particular, the use of point-splitting techniques leads to expressions that contain the van Vleck determinant as a common prefactor. Recent technical advances permit one to undertake extensive computations of the van Vleck determinant in traversable wormhole spacetimes - at least in the short-throat flat-space approximation. This paper presents several such computations for various model spacetimes. Implications with regard to Hawking's chronology protection conjecture are discussed.

In particular, any attempt to transform a single isolated wormhole into a time machine results in large vacuum polarization effects. These vacuum polarization effects are sufficient to disrupt the internal structure of the wormhole long before the onset of Planck scale physics, and before the onset of time travel. Thus for isolated wormholes, vacuum polarization effects are sufficient to enforce Hawking's chronology protection conjecture.
\end{abstract}

On the other hand, it is possible to conceive of a putative time machine built out of two or more wormholes, each of which taken in isolation is not 
itself a time machine. Such "Roman configurations" are much more subtle to analyse. For "reasonable" configurations (traversable by humans) the vacuum polarization effects in such multiple wormhole putative time machines become large long before the onset of Planck scale physics. The disruption scale for would be "traversable time machines" is well above the Planck scale. On the other hand, for some particularly bizarre configurations (not traversable by humans) the vacuum polarization effects can be arranged to be arbitrarily small at the onset of Planck scale physics. This indicates that the disruption scale has been pushed down into the Planck slop. This is mildly disturbing. Ultimately, for these bizarre configurations, questions regarding the truth or falsity of Hawking's chronology protection can only be addressed by entering the uncharted wastelands of full fledged quantum gravity.

04.20.-q, 04.20.Cv, 04.60.+n

Typeset using REVTEX 


\section{INTRODUCTION}

Hawking's recently formulated chronology protection conjecture [1,2] has prompted a considerable amount of activity. That conjecture, and various other issues regarding the existence and putative stability of traversable wormholes [3] 6], have led several authors to present calculations of the vacuum expectation value of the quantum stress-energy tensor in various spacetimes containing traversable wormholes [7 [12]. Generically one encounters expressions of the form

$$
\left\langle 0\left|T_{\mu \nu}(x)\right| 0\right\rangle \approx \hbar \sum_{\gamma}^{\prime} \frac{\Delta_{\gamma}(x, x)^{1 / 2}}{\pi^{2} s_{\gamma}(x, x)^{4}} t_{\mu \nu}(x ; \gamma)
$$

The sum runs over all nontrivial geodesics connecting the point $x$ to itself. The symbol $s_{\gamma}(x, x)$ denotes the length of the aforementioned geodesic. The tensor $t_{\mu \nu}(x ; \gamma)$ is a dimensionless object that is built up out of the spacetime metric and the tangent vectors to

$\gamma$ [10]. Furthermore $\Delta_{\gamma}(x, x)$ denotes the van Vleck determinant [13 19]. The overall size of this prefactor is a key ingredient in governing whether or not semiclassical quantum effects are sufficient to enforce Hawking's chronology protection conjecture [1, 2, 0,9 12]. To get a handle on what is going on, note that a good parameterization of the magnitude of the quantum back reaction is obtained by considering the scalar invariant

$$
\mathcal{T} \equiv \sqrt{\left\langle 0\left|T_{\mu \nu}(x)\right| 0\right\rangle\left\langle 0\left|T^{\mu \nu}(x)\right| 0\right\rangle}
$$

Then, if one of the closed self linking geodesics (say $\gamma^{\prime}$ ) dominates over the others, one may approximate

$$
\mathcal{T} \approx \hbar \frac{\Delta_{\gamma^{\prime}}(x, x)^{1 / 2}}{\pi^{2} s_{\gamma^{\prime}}(x, x)^{4}}
$$

The essential point is that the semiclassical quantum back reaction is directly proportional to the square root of the van Vleck determinant. While the above formula is valid at any point $x$ in the spacetime, it is most useful to consider a point $x$ on the throat of the wormhole. The size of the back reaction should be compared to the quantity of stress-energy required 
to hold the wormhole throat open in the first place. At the throat of a wormhole whose mouth is of radius $R$ the Einstein equations imply

$$
\mathcal{T}_{0} \equiv \sqrt{T_{\mu \nu} T^{\mu \nu}} \approx \frac{\hbar}{\ell_{P}^{2} R^{2}} .
$$

Once $\mathcal{T} \gg \mathcal{T}_{0}$ one has what I feel is clear and convincing evidence that the semiclassical vacuum polarization disrupts the internal structure of the wormhole [10]. (Other authors may choose to disagree with me on this issue). This disruption occurs at

$$
\left.s\right|_{\text {disrupt }} \approx \Delta_{\gamma^{\prime}}^{1 / 8} \sqrt{\pi \ell_{P} R} .
$$

Since $s_{\gamma}(x, x) \rightarrow 0$ is the signal for the onset of time travel, it will always be the case that this occurs before the time machine has a chance to form. The real issue is whether the overwhelming of the wormhole's internal structure occurs before or after one reaches the Planck regime.

If $\mathcal{T} \gg \mathcal{T}_{0}$ while $s_{\gamma}(x, x) \gg \ell_{P}$, then semiclassical vacuum polarization effects overwhelm the wormhole's internal structure in a parameter regime where one still expects the semiclassical approximation to hold. One may thus safely assert that at least in this parameter regime, semiclassical vacuum polarization effects enforce Hawking's chronology protection conjecture.

If $\mathcal{T} \gg \mathcal{T}_{0}$ does not occur until $s_{\gamma}(x, x)<\ell_{P}$, then one cannot conclude that time machine formation succeeds. All that one can safely conclude is that the uncharted wastelands of full fledged quantum gravity have been entered.

A central issue in investigating these issues is thus the evaluation of $\Delta_{\gamma}(x, x)$ for closed geodesics $\gamma$ with base point $x$ located on the throat of the wormhole. In fact, I shall try to be more general than that.

Recent technical advances [20] permit radical improvements in the computation of the van Vleck determinant. Basic tools employed are the reformulation of the van Vleck determinant in terms of tidal focussing effects; and consequent formal expansion for the van Vleck determinant in terms of the Riemann tensor. If the Riemann curvature is confined to 
relatively thin layers a suitable extension of the usual thin-shell formalism permits reduction of this formal expansion to a finite number of terms. The number $N$ of terms occurring in this expansion is just the number of times the closed geodesic $\gamma$ intersects a layer of high curvature. Several variations on this theme are presented.

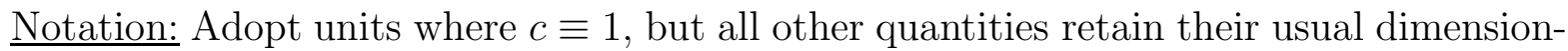
alities. In particular $G=\hbar / m_{P}^{2}=\ell_{P}^{2} / \hbar$. The metric signature is taken to be $(-,+,+,+)$. General conventions follow Misner, Thorne, and Wheeler 21.

\section{THE VAN VLECK DETERMINANT}

The primary definition of the (scalarized) van Vleck determinant is in terms of the second derivatives of the arc-length function

$$
\Delta_{\gamma}(x, y) \equiv \pm \frac{1}{2} \frac{1}{\sqrt{g(x) g(y)}} \operatorname{det}\left\{\frac{\partial^{2}\left[s_{\gamma}(x, y)\right]^{2}}{\partial x^{\mu} \partial y^{\nu}}\right\} .
$$

The symbol $s_{\gamma}(x, y)$ denotes the arc-length along the geodesic $\gamma$.

In a Lorentzian spacetime (or a Riemannian manifold) the van Vleck determinant can be reformulated in terms of tidal focussing effects [20]. Define

$$
\Delta_{\gamma}(x, y)=\operatorname{det}\left[s_{\gamma}(x, y) A^{-1}\right]
$$

Here the matrix $A$ describes the evolution of a set of Jacobi fields along the geodesic $\gamma$. In

terms of components defined by Fermi-Walker transporting a set of basis vectors along the geodesic $\gamma$, the matrix $A$ is governed by the second-order differential equation [20,22,

$$
\frac{d^{2}}{d s^{2}} A_{\nu}^{\mu}(s)=-\left(R_{\alpha \sigma \beta}^{\mu} t^{\alpha} t^{\beta}\right) A_{\nu}^{\sigma}
$$

The relevant boundary conditions are that $A^{\mu}{ }_{\nu}(s) \rightarrow \delta^{\mu}{ }_{\nu}$ as $s \rightarrow 0$. Introducing the onedimensional Green function $G_{R}\left(s_{f}, s_{i}\right)=\left\{s_{f}-s_{i}\right\} \Theta\left(s_{f}-s_{i}\right)$, this may be reformulated as an integral equation

$$
A_{\nu}^{\mu}(s)=s \delta_{\nu}^{\mu}-\int_{0}^{s} G_{R}\left(s, s^{\prime}\right)\left(R^{\mu}{ }_{\alpha \sigma \beta} t^{\alpha} t^{\beta}\right) A^{\sigma}{ }_{\nu}\left(s^{\prime}\right) d s^{\prime} .
$$


This integral equation may be solved by iteration. Defining $Q^{\mu}{ }_{\nu} \equiv-\left(R_{\alpha \nu \beta}^{\mu} t^{\alpha} t^{\beta}\right)$, and suppressing the explicit integration by regarding $G_{R}\left(s, s^{\prime}\right)$, multiplication by $Q(s)$, and multiplication by $s$, as functional operators

$$
A=\left(I+\left[G_{R} Q\right]+\left[G_{R} Q\right]^{2}+\cdots\right)\{s I\}
$$

The formalism as presented here applies equally well to spacelike or timelike geodesics. Lightlike geodesics require a little extra technical fiddling — for details see reference [20].

\section{WORMHOLE GEOMETRY: FIRST APPROXIMATION}

Consider a traversable wormhole: Assume that the throat of the wormhole is very short, and that curvature in the region outside the mouth of the wormhole is relatively weak. Such a wormhole can be idealised by considering Minkowski space with two regions excised, and then identifying the boundaries of those regions in some suitable manner. The Riemann tensor for such an idealised geometry is identically zero everywhere except at the wormhole

mouths/throat where the identification procedure takes place [5,6]. Generically, there will be an infinitesimally thin layer of exotic matter present at the mouth of the wormhole [3, 4 .

\section{A. First approximation: first attempt}

Consider now a geodesic that wraps through the mouth of the wormhole a total of $N$ times. In this short-throat flat-space approximation one has

$$
Q_{\nu}^{\mu}(s) \equiv-\left(R_{\alpha \nu \beta}^{\mu} t^{\alpha} t^{\beta}\right)=\sum_{n=1}^{N} \delta\left(s-s_{n}\right)\left[q(n)^{\mu}{ }_{\nu}\right] .
$$

This greatly simplifies the various terms in the expansion for the van Vleck determinant. In particular

$$
\left[G_{R} Q\right]\{s I\}=\sum_{n=1}^{N}\left(s-s_{n}\right)\left[q(n)^{\mu}{ }_{\nu}\right] s_{n}
$$

Furthermore 


$$
\left[G_{R} Q\right]^{2}\{s I\}=\sum_{n=2}^{N} \sum_{m=1}^{n-1}\left(s-s_{n}\right)\left[q(n)_{\rho}^{\mu}\right]\left(s_{n}-s_{m}\right)\left[q(m)^{\mu}{ }_{\nu}\right] s_{m} .
$$

While, using the symbol $\bullet$ to denote a generic dummy index,

$$
\begin{gathered}
{\left[G_{R} Q\right]^{N-1}\{s I\}=\sum_{n=1}^{N}\left(s-s_{N}\right)\left[q(N)^{\mu} \bullet\right]\left(s_{N}-s_{N-1}\right) \cdots} \\
{\left[q(n+1)^{\bullet} \bullet\right]\left(s_{n+1}-s_{n-1}\right)\left[q(n-1)^{\bullet} \bullet\right]} \\
\left(s_{2}-s_{1}\right)\left[q(1)^{\bullet}{ }_{\nu}\right] s_{1} .
\end{gathered}
$$

Ultimately

$$
\left[G_{R} Q\right]^{N}\{s I\}=\left(s-s_{N}\right)\left[q(N)^{\mu} \bullet\right]\left(s_{N}-s_{N-1}\right) \cdots\left(s_{2}-s_{1}\right)\left[q(1)^{\bullet}{ }_{\nu}\right] s_{1} .
$$

Due to the presence of a sufficient number of Heavyside functions, higher powers of $\left[G_{R} Q\right]$ vanish: $\left[G_{R} Q\right]^{N+n}\{s I\} \Rightarrow 0$ for $n>0$. Note that the formal expansion (10) now terminates in a finite number of steps

$$
A=\left(I+\left[G_{R} Q\right]+\left[G_{R} Q\right]^{2}+\cdots+\left[G_{R} Q\right]^{N}\right)\{s I\} .
$$

Here $N$ denotes the total number of trips through the wormhole. Observe, either from the above, or from the differential equation (8), that in this type of geometry the matrix $A(s)$ is a piecewise linear continuous matrix function of arc length. Consequently the reciprocal of the van Vleck determinant $\Delta_{\gamma}(s)^{-1}=\operatorname{det}\{A / s\}$ is piecewise a Laurent polynomial in arc length [20].

At this stage, I am deliberately leaving the matrices $\left[q(n)^{\mu}{ }_{\nu}\right]$ as general as possible so as to obtain results that are to a large extent independent of the particular details of the shape of the wormhole mouths and/or the identification procedure adopted at the wormhole mouths. Suitable specializations will be introduced in due course.

\section{B. First approximation: single pass}

For a geodesic that makes only a single pass through the mouth of the wormhole $(N=1)$, one easily derives an exact closed form expression 


$$
A^{\mu}{ }_{\nu}(s)=s \delta^{\mu}{ }_{\nu}+\Theta\left(s-s_{1}\right)\left\{\left(s-s_{1}\right)\left[q(1)^{\mu}{ }_{\nu}\right] s_{1}\right\}
$$

This result may be obtained either from the formal manipulations of the preceding section, or from direct integration of the tidal equation. This leads to a closed form for the van Vleck determinant. For $s \geq s_{1}$ :

$$
\Delta_{\gamma}(s)^{-1}=\operatorname{det}\left(\delta^{\mu}{ }_{\nu}+\frac{\left(s-s_{1}\right) s_{1}}{s}\left[q(1)^{\mu}{ }_{\nu}\right]\right)
$$

Particularize to a closed geodesic, so that $x=y$. Define $s_{+}=s_{1}, s_{-}=s-s_{1}$, and let $s=s_{+}+s_{-}$denote the total arc length. For convenience, one may set $\left[q^{\mu}{ }_{\nu}\right] \equiv\left[q(1)^{\mu}{ }_{\nu}\right]$. For such a closed geodesic

$$
\Delta_{\gamma}(x, x)=\operatorname{det}\left(\delta^{\mu}{ }_{\nu}+\frac{s_{+} s_{-}}{s}\left[q^{\mu}{ }_{\nu}\right]\right)^{-1}
$$

Suppose that the point of interest, $x=y$, lies near the throat of the wormhole. Then either $s_{+} \approx 0$ or $s_{-} \approx 0$.

Lemma: For any closed geodesic $\gamma$ that threads the throat of the wormhole once, for any point $x$ near the throat of the wormhole, the short-throat flat-space approximation yields:

$$
\Delta_{\gamma}(x, x)=1-\frac{s_{+} s_{-}}{s} \operatorname{tr}[q]+O\left[s_{+}^{2} s_{-}^{2} / s^{2}\right]
$$

At the throat itself, of course, this implies $\Delta_{\gamma}(x, x)=1$. This observation is already of some interest. See [10] and the discussion section of this paper. This result is compatible with, and a generalization of, the $N=1$ case of equation (48) of Kim and Thorne [7].

On the other hand, suppose that the point $x=y$ is far away from the wormhole throat. The behaviour of the van Vleck determinant is now governed by whether or not the eigenvalues of $\left\{s_{+} s_{-} / s\right\}\left[q^{\mu}{ }_{\nu}\right]$ ever become large compared to 1 . This depends on the details of the wormhole's construction.

Lemma: For any closed geodesic $\gamma$ that threads the throat of the wormhole once, for any point $x$ far away from throat of the wormhole, the short-throat flat-space approximation yields: 


$$
\Delta_{\gamma}(x, x)=\left[\frac{s}{s_{+} s_{-}}\right]^{\#} \operatorname{det}^{\prime}[q]^{-1}+O\left[\left(\frac{s}{s_{+} s_{-}}\right)^{\#-1}\right] .
$$

Here \# denotes the number of large eigenvalues of the matrix $\left\{s_{+} s_{-} / s\right\}\left[q^{\mu}{ }_{\nu}\right]$. Furthermore det $^{\prime}$ denotes the determinant with small eigenvalues omitted. If all of the eigenvalues are small, then $\#=0, \operatorname{det}^{\prime}[q]=1$, and consequently $\Delta_{\gamma}(x, x) \approx 1$.

This is compatible with the $N=1$ case of equation (64) of Kim and Thorne [0]. The present result is derived in a much more general context than their result but does not (yet) contain as much information.

\section{First approximation: double pass}

For a geodesic that makes two passes through the mouth of the wormhole $(N=2)$, one has the closed form expression

$$
\begin{aligned}
A^{\mu}{ }_{\nu}(s)= & s \delta^{\mu}{ }_{\nu} \\
& +\Theta\left(s-s_{1}\right)\left\{\left(s-s_{1}\right)\left[q(1)^{\mu}{ }_{\nu}\right] s_{1}\right\} \\
& +\Theta\left(s-s_{2}\right)\left\{\left(s-s_{2}\right)\left[q(2)^{\mu}{ }_{\nu}\right] s_{2}\right\} \\
& +\Theta\left(s-s_{2}\right)\left\{\left(s-s_{2}\right)\left[q(2)^{\mu}{ }_{\rho}\right]\left(s_{2}-s_{1}\right)\left[q(1)^{\rho}{ }_{\nu}\right] s_{1}\right\} .
\end{aligned}
$$

As was the case previously, this result may be derived either from the formal expansion, or from direct integration of the tidal equation.

Now particularize to a closed geodesic, so that $x=y$. Define $s_{+}=s_{1}, s_{-}=s-s_{2}$, and $s_{0}=s_{2}-s_{1}$. Then $s=s_{+}+s_{0}+s_{-}$denotes the total arc length. For convenience, one may set $\left[q_{+}\right]=[q(1)]$, and $\left[q_{-}\right]=[q(2)]$. Thus, for a closed two-pass geodesic

$$
\Delta_{\gamma}(x, x)^{-1}=\operatorname{det}\left(\delta^{\mu}{ }_{\nu}+\frac{s_{+}\left(s_{-}+s_{0}\right)}{s}\left[q_{+\nu}^{\mu}\right]+\frac{s_{-}\left(s_{+}+s_{0}\right)}{s}\left[q_{-\nu}^{\mu}\right]+\frac{s_{+} s_{-} s_{0}}{s}\left[q_{-\rho}^{\mu}\right]\left[q_{+\nu}^{\rho}\right]\right) .
$$

Now suppose the base point $x$ of the geodesic is near the throat of the wormhole. For definiteness take $s_{+} \equiv s_{1} \approx 0$, though one could just as easily take $s_{-} \equiv s-s_{2} \approx 0$. Introduce some new notation: $\epsilon \equiv s_{1} \approx 0, s_{1 \rightarrow 2} \equiv s_{2}-s_{1}, s_{2 \rightarrow 1} \equiv s_{1}+s-s_{2} \approx s-s_{2}$. 
Lemma: For any closed geodesic $\gamma$ that threads the throat of the wormhole twice, for any point $x$ near the throat of the wormhole, the short-throat flat-space approximation yields:

$$
\Delta_{\gamma}(x, x)^{-1}=\operatorname{det}\left(\delta^{\mu}{ }_{\nu}+\frac{s_{1 \rightarrow 2} s_{2 \rightarrow 1}}{s_{1 \rightarrow 2}+s_{2 \rightarrow 1}}\left[q(2)^{\mu}{ }_{\nu}\right]+O[\epsilon]\right) .
$$

Taking \# to denote the number of large eigenvalues of the matrix $\frac{s_{1 \rightarrow 2} s_{2 \rightarrow 1}}{s_{1 \rightarrow 2}+s_{2} \rightarrow 1}\left[q(2)^{\mu}{ }_{\nu}\right]$ one may approximate

$$
\Delta_{\gamma}(x, x)^{-1} \approx\left[\frac{s_{1 \rightarrow 2} s_{2 \rightarrow 1}}{s_{1 \rightarrow 2}+s_{2 \rightarrow 1}}\right]^{\#} \operatorname{det}^{\prime}\left[q(2)^{\mu}{ }_{\nu}\right] .
$$

Far away from the wormhole throat the analysis is considerably more delicate. The critical question in this case is whether or not any of the eigenvalues of the matrix $\left\{\left(s_{+} s_{-} s_{0}\right) / s\right\}\left[q_{-} q_{+}\right]$are large.

Lemma: For any closed geodesic $\gamma$ that threads the throat of the wormhole twice, if any of these eigenvalues are large, the short-throat flat-space approximation yields:

$$
\Delta_{\gamma}(x, x)=\left[\frac{s}{s_{+} s_{-} s_{0}}\right]^{\#} \operatorname{det}^{\prime}\left[q_{+} q_{-}\right]^{-1}+O\left[\left(\frac{s}{s_{+} s_{-} s_{0}}\right)^{\#-1}\right] .
$$

As before, let \# denote the number of large eigenvalues, this time of the matrix $\left\{\left(s_{+} s_{-} s_{0}\right) / s\right\}\left[q_{+} q_{-}\right]$. If all of the eigenvalues are small, then one must revert to

$$
\Delta_{\gamma}(x, x)^{-1} \approx \operatorname{det}\left(\delta^{\mu}{ }_{\nu}+\frac{s_{+}\left(s_{-}+s_{0}\right)}{s}\left[q_{+\nu}^{\mu}\right]+\frac{s_{-}\left(s_{+}+s_{0}\right)}{s}\left[q_{-\nu}^{\mu}\right]\right) .
$$

For the case of a double pass through the wormhole, this is about as far as this general type of analysis can profitably be carried. To improve and extend the calculations one needs more detailed information about the matrices $[q(n)]$.

\section{First approximation: multiple passes}

If one were to retain a completely general geodesic, the algebraic complexity involved in evaluating the van Vleck determinant would quickly rise from the merely cumbersome to the absolutely prohibitive. Accordingly, for this section only, I shall specialize to the case of a completely smooth closed geodesic, that wraps around the wormhole $N$ times. 
Because I have assumed complete smoothness, including smoothness at the base point $x$, the geodesic is a $N$-fold overlay of a smooth once around the wormhole geodesic. Thus the points where the geodesic intersects the wormhole mouth are all identical and one has

$$
\left[q^{\mu}{ }_{\nu}\right] \equiv\left[q(1)^{\mu}{ }_{\nu}\right]=\left[q(2)^{\mu}{ }_{\nu}\right]=\cdots=\left[q(N)^{\mu}{ }_{\nu}\right]
$$

Furthermore

$$
s_{0} \equiv s_{N}-s_{N-1}=\cdots=s_{3}-s_{2}=s_{2}-s_{1} .
$$

Finally, define $s_{+}=s_{1}$, and $s_{-}=s-s_{N}$. Since the total length of the geodesic is $s=N s_{0}$ it follows that $s_{+}+s_{-}=s_{0}$. Indeed $s_{n}=s_{+}+(n-1) s_{0}=n s_{0}-s_{-}$. Evaluating the various terms in the expansion for the van Vleck determinant will require a brief agony of combinatorics.

\section{Zero-order term}

The zeroth order term is just

$$
\left[G_{R} Q\right]^{0}\{s I\}=s=N s_{0}
$$

\section{First-order term}

It is a simple matter to see that

$$
\left[G_{R} Q\right]\{s I\}=\sum_{n=1}^{N}\left(s_{-}+[N-n] s_{0}\right)\left(s_{+}+[n-1] s_{0}\right) \times\left[q^{\bullet} \bullet\right] .
$$

Define $p_{ \pm}=s_{ \pm} / s_{0}$, so that $p_{+}+p_{-}=1$. Extracting all dimensionfull factors yields

$$
\left[G_{R} Q\right]\{s I\}=s_{0} S(N, 1)\left(s_{0}\left[q^{\bullet} \cdot\right]\right)
$$

The sum can be rearranged to be 


$$
\begin{aligned}
S(N, 1) & =\sum_{n=1}^{N}\left(p_{+} p_{-}+\left\{p_{+}[N-n]+p_{-}[n-1]\right\}+[N-n][n-1]\right) \\
& =\sum_{n=1}^{N}\left(p_{+} p_{-}+[N-n]+[N-n][n-1]\right) \\
& =\sum_{n=1}^{N}\left(p_{+} p_{-}+[N-n] n\right)
\end{aligned}
$$

Recall that $\sum_{n=1}^{N} n^{2}=N(N+1)(2 N+1) / 6$. A little work now gives

$$
\sum_{n=1}^{N}[N-n] n=\frac{(N-1) N(N+1)}{6}=\left(\begin{array}{c}
N+1 \\
3
\end{array}\right) .
$$

Collecting terms and rearranging

$$
\left[G_{R} Q\right]\{s I\}=s_{0}\left\{N p_{+} p_{-}+\left(\begin{array}{c}
N+1 \\
3
\end{array}\right)\right\}\left(s_{0}[q \bullet \bullet) .\right.
$$

\section{Second-order term}

The second-order term in the expansion is simply

$$
\left[G_{R} Q\right]^{2}\{s I\}=\sum_{n=2}^{N} \sum_{m=1}^{n-1}\left(s-s_{n}\right)\left(s_{n}-s_{m}\right) s_{m} \times[q \bullet \cdot]^{2} .
$$

Extracting all dimensionfull factors

$$
\left[G_{R} Q\right]^{2}\{s I\}=s_{0} S(N, 2)\left(s_{0}\left[q^{\bullet} \bullet\right]\right)^{2}
$$

The series summation is a trifle messy:

$$
\begin{aligned}
S(N, 2) & =\sum_{n=2}^{N} \sum_{m=1}^{n-1}\left(p_{-}+[N-n]\right)[n-m]\left(p_{+}+[m-1]\right), \\
& =\sum_{n=1}^{N} \sum_{m=1}^{n}\left(p_{-}+[N-n]\right)[n-m]\left(p_{+}+[m-1]\right) .
\end{aligned}
$$

Expanding the sum

$$
\begin{aligned}
S(N, 2)=\sum_{n=1}^{N} \sum_{m=1}^{n} & \left\{p_{+} p_{-}[n-m]\right. \\
& +p_{+}([N-n][n-m]) \\
& +p_{-}([n-m][m-1]) \\
& +[N-n][n-m][m-1]\} .
\end{aligned}
$$


The terms linear in $p_{+}$and $p_{-}$have identical coefficients. They may be combined using $p_{+}+p_{-}=1$, and can then be collapsed onto the constant term. This can be seen by noting

$$
\begin{aligned}
\sum_{n=1}^{N} \sum_{m=1}^{n}[N-n][n-m] & =\sum_{m=1}^{N} \sum_{n=m}^{N}[N-n][n-m] \\
& =\sum_{m=1}^{N} \sum_{n=1}^{N-m+1}[N-m+1-n][n-1] \\
& =\sum_{m=1}^{N} \sum_{n=1}^{m}[m-n][n-1] \\
& =\sum_{n=1}^{N} \sum_{m=1}^{n}[n-m][m-1] .
\end{aligned}
$$

This reduces the summation to

$$
S(N, 2)=\sum_{n=1}^{N} \sum_{m=1}^{n}\left\{p_{+} p_{-}[n-m]+[N-n][n-m] m\right\}
$$

Note

$$
\begin{aligned}
\sum_{n=1}^{N} \sum_{m=1}^{n}[n-m] & =\sum_{n=1}^{N}\left[n^{2}-\frac{n(n+1)}{2}\right] \\
& =\sum_{n=1}^{N} \frac{n(n-1)}{2} \\
& =\frac{(N-1) N(N+1)}{6}=\left(\begin{array}{c}
N+1 \\
3
\end{array}\right) .
\end{aligned}
$$

To evaluate the remaining term in $S(N, 2)$, recall the identities

$$
\left(\begin{array}{l}
n+1 \\
k+1
\end{array}\right)=\left(\begin{array}{l}
n \\
k
\end{array}\right)+\left(\begin{array}{c}
n \\
k+1
\end{array}\right)
$$

and

$$
\sum_{n=1}^{N}\left(\begin{array}{l}
n \\
k
\end{array}\right)=\sum_{n=k}^{N}\left(\begin{array}{l}
n \\
k
\end{array}\right)=\left(\begin{array}{l}
N+1 \\
k+1
\end{array}\right)
$$

Simple but tedious manipulations now give

$$
\begin{aligned}
\sum_{n=1}^{N} \sum_{m=1}^{n}[N-n][n-m] m & =\sum_{n=1}^{N}[N-n]\left(\begin{array}{c}
n+1 \\
3
\end{array}\right) \\
& =\sum_{n=1}^{N}[(N+2)-(n+2)]\left(\begin{array}{c}
n+1 \\
3
\end{array}\right)
\end{aligned}
$$




$$
\begin{aligned}
& =\sum_{n=1}^{N}\left\{[N+2]\left(\begin{array}{c}
n+1 \\
3
\end{array}\right)-4\left(\begin{array}{c}
n+2 \\
4
\end{array}\right)\right\} \\
& =[N+2]\left(\begin{array}{c}
N+2 \\
4
\end{array}\right)-4\left(\begin{array}{c}
N+3 \\
5
\end{array}\right) \\
& =\left(\begin{array}{c}
N+2 \\
5
\end{array}\right) .
\end{aligned}
$$

So finally

$$
S(N, 2)=\left(\begin{array}{c}
N+1 \\
3
\end{array}\right) p_{+} p_{-}+\left(\begin{array}{c}
N+2 \\
5
\end{array}\right)
$$

The beginning of a pattern might be discerned from this result.

$$
\text { 4. n'th-order term }
$$

The general n'th-order term is

$$
\begin{aligned}
{\left[G_{R} Q\right]^{n}\{s I\}=\sum_{m_{n}=}^{N} } & \sum_{m_{n-1}=n-1}^{m_{n}} \sum_{m_{n-2}=n-2}^{m_{n-1}} \cdots \sum_{m_{3}=3}^{m_{4}} \sum_{m_{2}=2}^{m_{3}} \sum_{m_{1}=1}^{m_{2}} \\
& \left(s-s_{m_{n}}\right)\left(s_{m_{n}}-s_{m_{n-1}}\right) \cdots\left(s_{m_{2}}-s_{m_{1}}\right) s_{m_{1}} \\
& \times\left[q^{\bullet} \bullet\right]^{n} .
\end{aligned}
$$

Extracting all dimensionfull factors

$$
\left[G_{R} Q\right]^{n}\{s I\}=s_{0} S(N, n)\left(s_{0}\left[q^{\bullet} \bullet \cdot\right]\right)^{n} .
$$

The relevant series sum is

$$
\begin{aligned}
S(N, n) \equiv \sum_{m_{n}=n}^{N} \sum_{m_{n-1}=n-1}^{m_{n}} \sum_{m_{n-1}=n-2}^{m_{n-1}} \cdots \sum_{m_{3}=3}^{m_{4}} \sum_{m_{2}=2}^{m_{3}} \sum_{m_{1}=1}^{m_{2}} \\
\quad\left(p_{-}+\left[N-m_{n}\right]\right)\left(m_{n}-m_{n-1}\right) \cdots\left(m_{2}-m_{1}\right)\left(p_{+}+\left[m_{1}-1\right]\right) .
\end{aligned}
$$

This has the form

$$
S(N, n)=j(N, n) p_{+} p_{-}+k^{+}(N, n) p_{+}+k^{-}(N, n) p_{-}+l(N, n) .
$$

Here 


$$
\begin{aligned}
k^{+}(N, n) \equiv \sum_{m_{n}=n}^{N} \sum_{m_{n-1}=m_{m_{2}}}^{m_{n}} \sum_{m_{n-1}=1}^{n-1} \cdots \sum_{m_{3}=3}^{m_{4}} \sum_{m_{2}=2}^{m_{3}} \sum_{m_{1}=1}^{m_{2}} \\
\quad\left(N-m_{n}\right)\left(m_{n}-m_{n-1}\right) \cdots\left(m_{3}-m_{2}\right)\left(m_{2}-m_{1}\right) \\
=\sum_{m=n}^{N}[N-m] k^{+}(m, n) .
\end{aligned}
$$

On the other hand, $k^{-}(N, n)$ satisfies

$$
\begin{aligned}
k^{-}(N, n) & \equiv \sum_{m_{n}=n}^{N} \sum_{m_{n-1}=n-1}^{m_{n}} \sum_{m_{n-2}=n-2}^{m_{n-1}} \cdots \sum_{m_{3}=3}^{m_{4}} \sum_{m_{2}=2}^{m_{3}} \sum_{m_{1}=1}^{m_{2}} \\
& =\sum_{m_{1}=1}^{N-n} \sum_{m_{2}=m_{1}} \sum_{m_{3}=m_{2}}^{N-n+1} \cdots \sum_{m_{n-1}}^{N-n+2} \sum_{m_{n-3}} \sum_{m_{n-1}=m_{n-2}}^{N-1} \sum_{m_{n}=m_{n-1}}^{N} \\
& =\sum_{m=1}^{N-n} k^{-}\left(N-m_{n-2}\right) \cdots\left(m_{2}-m_{1}\right)\left(m_{1}-1\right) \\
= & \left.\sum_{m=n}^{N}[N-m] m_{n-1}\right)\left(m_{n-1}-m_{n-2}\right) \cdots\left(m_{2}-m_{1}\right)\left(m_{1}-1\right)
\end{aligned}
$$

So $k^{+}$and $k^{-}$satisfy the same recursion relation. But

$$
k^{+}(N, 1)=\sum_{m=1}^{N}(N-m)=\frac{N(N-1)}{2}=\sum_{m=1}^{N}(m-1)=k^{-}(N, 1) .
$$

This is enough to tell one that for all $(N, n), k^{+}(N, n)=k^{-}(N, n)$. One can actually do better than this by guessing the solution to the recursion equation and checking the result

$$
k^{+}(N, n)=k^{-}(N, n)=\left(\begin{array}{c}
N+n-1 \\
2 n
\end{array}\right)
$$

To prove this, note

$$
\begin{aligned}
\sum_{m=n}^{N}[N-m]\left(\begin{array}{c}
m+n-2 \\
2 n-2
\end{array}\right) & =\sum_{m=n}^{N}\{[N+n-1]-[m+n-1]\}\left(\begin{array}{c}
m+n-2 \\
2 n-2
\end{array}\right) \\
& =\sum_{m=n}^{N}\left\{[N+n-1]\left(\begin{array}{c}
m+n-2 \\
2 n-2
\end{array}\right)-[2 n-1]\left(\begin{array}{c}
m+n-1 \\
2 n-1
\end{array}\right)\right\} \\
& =[N+n-1]\left(\begin{array}{c}
N+n-1 \\
2 n-1
\end{array}\right)-[2 n-1]\left(\begin{array}{c}
N+n \\
2 n
\end{array}\right) \\
& =\left(\begin{array}{c}
N+n-1 \\
2 n
\end{array}\right) .
\end{aligned}
$$


It is now useful to define

$$
\ell(N, n) \equiv l(N, n)+k^{+}(N, n) \equiv l(N, n)+k^{-}(N, n)
$$

Explicitly

$$
\begin{aligned}
\ell(N, n) \equiv \sum_{m_{n}=n}^{N} \sum_{m_{n-1}=n-1}^{m_{n}} \sum_{m_{n-2}=n-2}^{m_{n-1}} \cdots \sum_{m_{3}=3}^{m_{4}} \sum_{m_{2}=2}^{m_{3}} \sum_{m_{1}=1}^{m_{2}} \\
\quad\left(N-m_{n}\right)\left(m_{n}-m_{n-1}\right) \cdots\left(m_{3}-m_{2}\right)\left(m_{2}-m_{1}\right) m_{1} \\
=\sum_{m=n}^{N}[N-m] \ell(m, n-1) .
\end{aligned}
$$

From previous computations one already knows $\ell(N, 1)=\left(\begin{array}{c}N+1 \\ 3\end{array}\right)$, and $\ell(N, 2)=\left(\begin{array}{c}N+2 \\ 5\end{array}\right)$. Minor modifications of the technique used in evaluating $k^{ \pm}(N, n)$ shows

$$
\ell(N, n)=\left(\begin{array}{c}
N+m \\
2 n+1
\end{array}\right)
$$

Finally, turn attention to the quantity

$$
\begin{aligned}
j(N, n) \equiv & \sum_{m_{n}=n}^{N} \sum_{m_{n-1}=n-1}^{m_{n}} \sum_{m_{n-2}=n-2}^{m_{n-1}} \cdots \sum_{m_{3}=3}^{m_{4}} \sum_{m_{2}=2}^{m_{3}} \sum_{m_{1}=1}^{m_{2}} \\
& \quad\left(m_{n}-m_{n-1}\right)\left(m_{n-1}-m_{n-2}\right) \cdots\left(m_{3}-m_{2}\right)\left(m_{2}-m_{1}\right) \\
= & \sum_{m=n}^{N} k^{+}(m, n-1) \\
= & \sum_{m=n}^{N}\left(\begin{array}{c}
m+n-2 \\
2 n-2
\end{array}\right) \\
= & \left(\begin{array}{c}
N+n-1 \\
2 n-1
\end{array}\right) .
\end{aligned}
$$

Combining everything, one obtains the rather simple and pleasing result

$$
S(N, n)=\left(\begin{array}{c}
N+n-1 \\
2 n-1
\end{array}\right) p_{+} p_{-}+\left(\begin{array}{c}
N+n \\
2 n+1
\end{array}\right) .
$$

The result is in fact so simple that one suspects that there should be easier ways of deriving it. I reiterate the general n'th order term:

$$
\left[G_{R} Q\right]^{n}\{s I\}=s_{0}\left\{\left(\begin{array}{c}
N+n-1 \\
2 n-1
\end{array}\right) p_{+} p_{-}+\left(\begin{array}{c}
N+n \\
2 n+1
\end{array}\right)\right\}\left(s_{0}\left[q^{\bullet} \cdot \mathbf{\bullet}\right]\right)^{n} .
$$




\section{5. (N-1)'th-order term}

The (N-1)'th term in the expansion is relatively simple to write down explicitly

$$
\begin{aligned}
{\left[G_{R} Q\right]^{N-1}\{s I\}=} & \left(s_{-}+s_{0}\right)\left(s_{0}\right)^{N-2} s_{+} \\
& +\sum_{n=2}^{N-1} s_{-}\left(s_{0}\right)^{N-(n+1)}\left(2 s_{0}\right)\left(s_{0}\right)^{(n-1)-1} s_{+} \\
& \left.+s_{-}\left(s_{0}\right)^{N-2}\left(s_{+}+s_{0}\right)\right\}\left[q^{\bullet} \bullet\right]^{N-1} \\
= & \left\{2(N-1) \frac{s_{+} s_{-}}{s_{0}}+s_{0}\right\}\left(s_{0}\left[q^{\bullet} \bullet\right]\right)^{N-1} \\
= & s_{0}\left\{2(N-1) p_{+} p_{-}+1\right\}\left(s_{0}\left[q^{\bullet} \bullet\right)^{N-1} .\right.
\end{aligned}
$$

This result serves as a check on the general expression for the n'th order term.

\section{N'th-order term}

Ultimately

$$
\left[G_{R} Q\right]^{N}\{s I\}=s_{-} s_{0}^{N-1} s_{+}\left[q^{\bullet} \bullet\right]^{N}=s_{0} p_{+} p_{-}\left(s_{0}\left[q^{\bullet} \bullet\right]\right)^{N}
$$

Again, this serves as a check on the general expression for the n'th order term.

\section{Collecting terms}

One is now in a position to write down a closed form expression for the van Vleck determinant

$$
\begin{aligned}
\Delta_{\gamma}^{-1} & =\operatorname{det}\left[s_{\gamma}(x, y)^{-1} A(s)\right] \\
& =\operatorname{det}\left[s^{-1}\left(I+\left[G_{R} Q\right]+\left[G_{R} Q\right]^{2}+\cdots+\left[G_{R} Q\right]^{N}\right)\{s I\}\right] \\
& =\operatorname{det}\left[\sum_{n=0}^{N} S(N, n)\left(s_{0}\left[q^{\bullet} \cdot\right]\right)^{n} / N\right] \\
& =\operatorname{det}\left[\left\{\left[I^{\bullet} \cdot\right]+p_{+} p_{-}\left(s_{0}\left[q^{\bullet} \bullet\right]\right)\right\} \times\right.
\end{aligned}
$$




$$
\begin{aligned}
&\left.\left\{\sum_{n=0}^{N-1}\left(\begin{array}{l}
N+n \\
2 n+1
\end{array}\right) \frac{\left(s_{0}\left[q^{\bullet} \cdot\right]\right)^{n}}{N}\right\}\right] \\
&=\operatorname{det}\left\{\left[I^{\bullet} \cdot\right]+p_{+} p_{-}\left(s_{0}\left[q^{\bullet} \cdot \mathbf{\cdot}\right]\right)\right\} \times \\
& \operatorname{det}\left\{\sum_{n=0}^{N-1}\left(\begin{array}{l}
N+n \\
2 n+1
\end{array}\right) \frac{\left(s_{0}\left[q^{\bullet} \cdot\right]\right)^{n}}{N}\right\} .
\end{aligned}
$$

This factorization property for the determinant is a quite remarkable and unexpected feature. Equally remarkable is the fact that the polynomials occurring in the above expression,

$$
P_{N}(z)=\sum_{n=0}^{N}\left(\begin{array}{c}
N+n+1 \\
2 n+1
\end{array}\right) z^{n}
$$

are nothing more than suitably disguised Chebyshev polynomials (a.k.a. Tschebischeff polynomials) of the second kind [23 25]. Indeed

$$
P_{N}(z)=U_{N}([z+4] / 2)
$$

(Since I will not need to use this result, I leave the proof as an exercise for the reader.)

Now, provided there is no accidental zero suppressing the highest order term, one may approximate

$$
\Delta_{\gamma}^{-1} \approx \operatorname{det}\left\{\left[I^{\bullet} \bullet\right]+p_{+} p_{-}\left(s_{0}\left[q^{\bullet} \bullet\right]\right)\right\} \operatorname{det}^{\prime}\left\{\frac{\left(s_{0}\left[q^{\bullet} \bullet\right]\right)^{N-1}}{N}\right\} .
$$

Far away from the mouth of the wormhole $p_{+} p_{-} \gg 0$, so that

$$
\Delta_{\gamma} \approx\left(\frac{N s_{0}^{2}}{s_{+} s_{-}}\right)^{\#} \operatorname{det}^{\prime}\left\{s_{0}\left[q^{\bullet} \bullet\right]\right\}^{-N} .
$$

This should be compared to eq. (64) of Kim and Thorne [7]. If the base point of the geodesic lies on the throat of the wormhole $p_{+} p_{-}=0$. The dominant term in the determinant is now

$$
\Delta_{\gamma} \approx N^{\#} \operatorname{det}^{\prime}\left\{s_{0}\left[q^{\bullet} \bullet\right]\right\}^{-N+1}
$$

Compare with eq (48) of Kim and Thorne [7]. A benefit of the current analysis is that it is now possible to probe the transition region as the base point of the geodesic approaches the throat of the wormhole. (i.e. $\left.s_{+} s_{-} \rightarrow 0\right)$.

Further simplifications require more precise model building for the wormhole in question. Useful models may be obtained by extending the usual thin-shell formalism (a.k.a. the junction condition formalism). 


\section{EXTENDED THIN SHELL FORMALISM: THEORY}

Development of the junction condition formalism, initiated by Lanczos [26] and Sen [27] in the early part of this century, culminated in the work of Israel [28], of Taub 229], and of Barrabes [30]. The Lanczos-Sen-Israel version of the formalism relates the Ricci tensor generated by the gravitational influence a thin shell of stress-energy to the discontinuity of the second fundamental forms defined by considering the thin shell to be an immersed submanifold of the full spacetime. Unfortunately, for the present application a knowledge of the Ricci tensor is not enough. An expression for the full Riemann tensor is necessary.

For the purposes of this paper, it is sufficient to consider a thin shell of stress-energy residing in an otherwise flat Lorentzian spacetime. Such a system is described by a number of copies of Minkowski space with two or more regions excised, and with the boundaries of those regions identified in some suitable manner. Examples of such systems include the traversable wormholes of interest in this paper (in the limit as the throat of the wormhole becomes arbitrarily short), and the cellular cosmologies of Redmount [31]. The Riemann tensor for such a geometry is identically zero everywhere except at the boundary where the identification procedure takes place. The formalism is presented for a timelike shell (spacelike normal). The modifications required to deal with a spacelike shell (timelike normal) are trivial. The general analysis for the case of a shell of null stress-energy is considerably more tedious, and will not be needed in this paper. The reader is referred to the work of Redmount [32] where an analysis in terms of the Newman-Penrose formalism is presented.

The modifications required in order to permit the wormhole to reside in a non-flat background are sufficiently simple that, in the interests of generality, the appropriate modifications are included. 


\section{A. Second fundamental form}

Consider a thin shell of stress-energy situated in some smooth (possibly curved) ambient space. Adopt Gaussian normal coordinates near the shell. That is: $\eta=0$ is the location of the shell, while $\eta$ itself is a normal coordinate. Without loss of generality the metric can, in the vicinity of the shell, be cast in the form

$$
g_{\alpha \beta}\left(\eta, x_{\perp}\right)=\Theta(\eta) g_{\alpha \beta}^{+}\left(\eta, x_{\perp}\right)+\Theta(-\eta) g_{\alpha \beta}^{-}\left(\eta, x_{\perp}\right) .
$$

The metric itself is continuous at the shell so that

$$
g_{\alpha \beta}^{+}\left(\eta=0, x_{\perp}\right)=g_{\alpha \beta}^{-}\left(\eta=0, x_{\perp}\right) .
$$

The second fundamental forms associated with the two sides of the shell $\eta=0$ are simply

$$
\begin{aligned}
& K_{\alpha \beta}^{+}=\left.\frac{1}{2} \frac{\partial g_{\alpha \beta}}{\partial \eta}\right|_{0^{+}}=\frac{1}{2} \frac{\partial g_{\alpha \beta}^{+}}{\partial \eta}, \\
& K_{\alpha \beta}^{-}=\left.\frac{1}{2} \frac{\partial g_{\alpha \beta}}{\partial \eta}\right|_{0^{-}}=\frac{1}{2} \frac{\partial g_{\alpha \beta}^{-}}{\partial \eta} .
\end{aligned}
$$

The discontinuity in the second fundamental form is

$$
\kappa_{\alpha \beta}=K_{\alpha \beta}^{+}-K_{\alpha \beta}^{-}
$$

while the normal to the thin shell satisfies

$$
n_{\alpha}=\partial_{\alpha} \eta ; \quad n^{\alpha} n_{\alpha}=+1 ; \quad n^{\alpha} \kappa_{\alpha \beta}=0
$$

Consider the metric derivatives $g_{\alpha \beta, \gamma}$. In view of the fact that $\frac{d}{d x} \Theta( \pm \eta)= \pm \delta(\eta)$, and of the continuity of the metric at $\eta=0$, one derives

$$
g_{\alpha \beta, \gamma}=\Theta(\eta) g_{\alpha \beta, \gamma}^{+}+\Theta(-\eta) g_{\alpha \beta, \gamma}^{-}
$$

It is now a simple application of Gaussian normal coordinates to show [26]

$$
g_{\alpha \beta, \gamma}^{+}-g_{\alpha \beta, \gamma}^{-}=2 \kappa_{\alpha \beta} n_{\gamma}
$$


Though most easily derived using Gaussian normal coordinates this result is general. The second derivatives of the metric are easily evaluated

$$
\begin{aligned}
g_{\alpha \beta, \gamma \delta} & =\Theta(\eta) g_{\alpha \beta, \gamma \delta}^{+}+\Theta(-\eta) g_{\alpha \beta, \gamma \delta}^{-}+\delta(\eta)\left\{g_{\alpha \beta, \gamma}^{+}-g_{\alpha \beta, \gamma}^{-}\right\} n_{\delta}, \\
& =\Theta(\eta) g_{\alpha \beta, \gamma \delta}^{+}+\Theta(-\eta) g_{\alpha \beta, \gamma \delta}^{-}+2 \delta(\eta) \kappa_{\alpha \beta} n_{\gamma} n_{\delta} .
\end{aligned}
$$

In a very similar vein, one may consider the Christoffel symbol

$$
\Gamma_{\alpha \beta \gamma} \equiv \frac{1}{2}\left[g_{\alpha \beta, \gamma}+g_{\alpha \gamma, \beta}-g_{\beta \gamma, \alpha}\right]=\Theta(\eta) \Gamma_{\alpha \beta \gamma}^{+}+\Theta(-\eta) \Gamma_{\alpha \beta \gamma}^{-} .
$$

\section{B. Riemann tensor}

With all of the preparatory work out of the way, it is now a simple matter of invoking the standard definition of the Riemann tensor

$$
\begin{aligned}
R_{\alpha \beta \gamma \delta}= & -\frac{1}{2}\left(g_{\alpha \gamma, \beta \delta}+g_{\beta \delta, \alpha \gamma}-g_{\alpha \delta, \beta \gamma}-g_{\beta \gamma, \alpha \delta}\right) \\
& -g^{\sigma \rho}\left(\Gamma_{\alpha \gamma \sigma} \Gamma_{\beta \delta \rho}-\Gamma_{\alpha \delta \sigma} \Gamma_{\beta \gamma \rho}\right),
\end{aligned}
$$

to see that the Riemann tensor in the vicinity of the thin shell is of the form

$$
\begin{gathered}
R_{\alpha \beta \gamma \delta}=-\delta(\eta)\left[\kappa_{\alpha \gamma} n_{\beta} n_{\delta}+\kappa_{\beta \delta} n_{\alpha} n_{\gamma}-\kappa_{\alpha \delta} n_{\beta} n_{\gamma}-\kappa_{\beta \gamma} n_{\alpha} n_{\delta}\right] \\
+\Theta(\eta) R_{\alpha \beta \gamma \delta}^{+}+\Theta(-\eta) R_{\alpha \beta \gamma \delta}^{-} .
\end{gathered}
$$

It is easy to see that the known symmetries of the Riemann tensor are correctly reflected in this expression. A little more work establishes that the full (uncontracted) Bianchi identities are satisfied. To check this, note that $\partial_{\alpha} \delta(\eta)=\delta^{\prime}(\eta) \partial_{\alpha} \eta=\delta^{\prime}(\eta) n_{\alpha}$, and that $\nabla_{\alpha} n_{\beta}=K_{\alpha \beta}^{ \pm}$is symmetric. The proof of the Bianchi identities follows by considering the antisymmetrization properties of $R_{\alpha \beta[\gamma \delta ; \epsilon]}$.

With hindsight, the above expression for the full Riemann tensor can be easily derived from standard textbook results. For instance, from equations (21.82), (21.75), and (21.76) of Misner, Thorne, and Wheeler [21] a gaussian pillbox integration similar to that discussed on pages 551 through 556 rapidly leads to the above result. 
By contraction on appropriate indices one may recover the known Lanczos-Sen-Israel junction conditions [26 30]. In terms of the Ricci tensor, the Einstein tensor, and the Ricci scalar:

$$
\begin{aligned}
R_{\alpha \beta} & =-\delta(\eta)\left[\kappa_{\alpha \beta}+\kappa n_{\alpha} n_{\beta}\right]+\Theta(\eta) R_{\alpha \beta}^{+}+\Theta(-\eta) R_{\alpha \beta}^{-} . \\
R & =-2 \kappa \delta(\eta)+\Theta(\eta) R^{+}+\Theta(-\eta) R^{-} . \\
E_{\alpha \beta} & =-\delta(\eta)\left[\kappa_{\alpha \beta}-\kappa\left(g_{\alpha \beta}-n_{\alpha} n_{\beta}\right)\right]+\Theta(\eta) E_{\alpha \beta}^{+}+\Theta(-\eta) E_{\alpha \beta}^{-} .
\end{aligned}
$$

These equations are completely equivalent to the usual Lanczos-Sen-Israel version of the junction condition formalism. Indeed, this is a quick consistency check.

\section{Jacobi tensor}

Tidal effects are sometimes reformulated in terms of the Jacobi tensor [21]

$$
\begin{aligned}
J_{\alpha \beta \gamma \delta} & \equiv \frac{1}{2}\left(R_{\alpha \gamma \beta \delta}+R_{\alpha \delta \beta \gamma}\right) \\
R_{\alpha \beta \gamma \delta} & \equiv \frac{2}{3}\left(J_{\alpha \gamma \beta \delta}+J_{\alpha \delta \beta \gamma}\right)
\end{aligned}
$$

This easily leads to

$$
\begin{aligned}
J_{\alpha \beta \gamma \delta}=-\delta( & \eta)\left[\kappa_{\alpha \beta} n_{\gamma} n_{\delta}+\kappa_{\gamma \delta} n_{\alpha} n_{\beta}\right. \\
& \left.-\frac{1}{2} \kappa_{\alpha \delta} n_{\beta} n_{\gamma}-\frac{1}{2} \kappa_{\beta \gamma} n_{\alpha} n_{\delta}-\frac{1}{2} \kappa_{\alpha \gamma} n_{\beta} n_{\delta}-\frac{1}{2} \kappa_{\beta \delta} n_{\alpha} n_{\gamma}\right] \\
& +\Theta(\eta) J_{\alpha \beta \gamma \delta}^{+}+\Theta(-\eta) J_{\alpha \beta \gamma \delta}^{-} .
\end{aligned}
$$

This result is, of course, completely equivalent to the result for the Riemann tensor.

\section{Weyl tensor}

The Weyl tensor describes the part of the curvature that is invariant under conformal rescalings of the metric. It may be computed by brute force starting from the definition 21,22, 


$$
\begin{aligned}
W_{\alpha \beta \gamma \delta} \equiv & +R_{\alpha \beta \gamma \delta} \\
& -\frac{1}{2}\left[g_{\alpha \gamma} R_{\beta \delta}+g_{\beta \delta} R_{\alpha \gamma}-g_{\alpha \delta} R_{\beta \gamma}-g_{\beta \gamma} R_{\alpha \delta}\right] \\
& +\frac{1}{12} R\left[g_{\alpha \gamma} g_{\beta \delta}+g_{\beta \delta} g_{\alpha \gamma}-g_{\alpha \delta} g_{\beta \gamma}-g_{\beta \gamma} g_{\alpha \delta}\right]
\end{aligned}
$$

To simplify life, define the transverse metric to be $h_{\alpha \beta} \equiv g_{\alpha \beta}-n_{\alpha} n_{\beta}$, and define the transverse

traceless part of the discontinuity in the second fundamental form to be $\tilde{\kappa}_{\alpha \beta} \equiv \kappa_{\alpha \beta}-\frac{1}{3} \kappa h_{\alpha \beta}$. The result of a little tedious algebra gives

$$
\begin{gathered}
W_{\alpha \beta \gamma \delta}=-\delta(\eta)\left[\tilde{\kappa}_{\alpha \gamma}\left(n_{\beta} n_{\delta}-\frac{1}{2} g_{\beta \delta}\right)+\tilde{\kappa}_{\beta \delta}\left(n_{\alpha} n_{\gamma}-\frac{1}{2} g_{\alpha \gamma}\right)\right. \\
\left.-\tilde{\kappa}_{\alpha \delta}\left(n_{\beta} n_{\gamma}-\frac{1}{2} g_{\beta \gamma}\right)-\tilde{\kappa}_{\beta \gamma}\left(n_{\alpha} n_{\delta}-\frac{1}{2} g_{\alpha \delta}\right)\right] \\
+\Theta(\eta) W_{\alpha \beta \gamma \delta}^{+}+\Theta(-\eta) W_{\alpha \beta \gamma \delta}^{-},
\end{gathered}
$$

which is easily verified to satisfy the relevant trace identities. As an aside, it is relatively easy to convince oneself that the tensors $\tilde{\kappa}_{\beta}^{\alpha} \equiv \kappa_{\beta}^{\alpha}-\frac{1}{3} \kappa h^{\alpha}{ }_{\beta}$, and $n^{\alpha} n_{\beta}$ are separately invariant under conformal transformations of the metric.

\section{E. Examples}

In the idealised case of a traversable wormhole constructed from flat Minkowski space by cut and paste techniques [5:6] one knows that the Riemann tensor must be zero everywhere except at the throat itself. So in this idealised case one obtains the exact result

$$
R_{\alpha \beta \gamma \delta}=-\delta(\eta)\left[\kappa_{\alpha \gamma} n_{\beta} n_{\delta}+\kappa_{\beta \delta} n_{\alpha} n_{\gamma}-\kappa_{\alpha \delta} n_{\beta} n_{\gamma}-\kappa_{\beta \gamma} n_{\alpha} n_{\delta}\right]
$$

Similar considerations apply to Redmount's cellular cosmologies [31]. In both cases one now has a very powerful tool for attacking problems involving tidal effects.

\section{EXTENDED THIN SHELL FORMALISM: APPLICATION}




\section{A. Riemann tensor}

It is possible to improve and make more explicit the general analysis of the van Vleck determinant by applying the extended thin-shell formalism as outlined above. For a wormhole in the short-throat flat-space approximation the full Riemann tensor is

$$
R_{\alpha \beta \gamma \delta}=-\delta(\eta)\left[\kappa_{\alpha \gamma} n_{\beta} n_{\delta}+\kappa_{\beta \delta} n_{\alpha} n_{\gamma}-\kappa_{\alpha \delta} n_{\beta} n_{\gamma}-\kappa_{\beta \gamma} n_{\alpha} n_{\delta}\right] .
$$

For the delta function one can easily show

$$
\delta(\eta)=(\partial \eta / \partial s)^{-1} \delta(s)=(t \cdot n)^{-1} \delta(s)
$$

The relevant source term driving the tidal evolution equation is

$$
\begin{aligned}
Q_{\mu \nu}(s) & \equiv-R_{\mu \alpha \nu \beta} t^{\alpha} t^{\beta} \\
& =\delta(\eta)\left[\kappa_{\mu \nu}(t \cdot n)^{2}+n_{\mu} n_{\nu}\left(\kappa_{\alpha \beta} t^{\alpha} t^{\beta}\right)-\left\{\left(\kappa_{\mu \alpha} t^{\alpha}\right) n_{\nu}+\left(\kappa_{\nu \alpha} t^{\alpha}\right) n_{\mu}\right\}(t \cdot n)\right] \\
& =\delta(s)\left[\kappa_{\mu \nu}(t \cdot n)+n_{\mu} n_{\nu}\left(\kappa_{\alpha \beta} t^{\alpha} t^{\beta}\right)(t \cdot n)^{-1}-\left\{\left(\kappa_{\mu \alpha} t^{\alpha}\right) n_{\nu}+\left(\kappa_{\nu \alpha} t^{\alpha}\right) n_{\mu}\right\}\right] .
\end{aligned}
$$

The discontinuity in the second fundamental form, $\kappa_{\mu \nu}$, is essentially a measure of the curvature of the wormhole mouth. Diagonalizing $\kappa$ yields $\kappa_{\mu \nu}=2 \operatorname{diag}\left\{A,\left(1 / R_{1}\right),\left(1 / R_{2}\right), 0\right\}_{\mu \nu}$. Here $A$ is the four-acceleration of the wormhole throat - essentially the radius of curvature in the timelike direction [6]. $R_{(1,2)}$ are the usual principal radii of curvature in the 3 dimensional sense. The zero eigenvalue for $\kappa$ reflects the fact that $\kappa_{\mu \nu} n^{\nu}=0$, the second fundamental form is by construction orthogonal to the normal.

One is now in a position to enunciate a general qualitative result:

(small radius of curvature/large acceleration) $\Rightarrow$ (large $\kappa) \Rightarrow$ (large Riemann tensor) $\Rightarrow$ (large $A(s)) \Rightarrow$ (small van Vleck determinant).

To proceed further, suppose that the individual wormhole mouths (though not the full spacetime) are static. This just means that the individual wormhole mouths are not un-

dergoing any changes in internal structure; and also implies that one can safely attach the notion of a constant four-velocity $V^{\mu}$ to each individual wormhole mouth. In particular 
since each wormhole mouth is now invariant under time translations along the $V^{\mu}$ axis one has $\kappa_{\mu \nu} V^{\nu}=0$. Further, by construction, $\kappa_{\mu \nu} n^{\nu}=0$ and $V^{\mu} n_{\mu}=0$. Indeed in this case $\kappa_{\mu \nu}=\operatorname{diag}\left\{0,\left(2 / R_{1}\right),\left(2 / R_{2}\right), 0\right\}_{\mu \nu}$. A brief computation shows $Q_{\mu \nu} V^{\nu}=0$. The general analysis already implies $Q_{\mu \nu} t^{\nu}=0$. Therefore, in this approximation two of the eigenvalues of $Q(s)$ are trivial, and the computation of the van Vleck determinant reduces to that of a (still decidedly non-trivial) $2 \times 2$ matrix.

\section{B. Single pass: exact result}

Consider a geodesic that starts at the point $x$, wraps through the wormhole once, and returns to the point $x$. This geodesic has two tangent vectors, $t_{ \pm}$, one pointing toward each of the two wormhole mouths. Each of the two wormhole mouths is characterized by a four-velocity $V_{ \pm}$, and, at the point where the geodesic impacts the mouth of the wormhole, a normal $n_{ \pm}$. In view of the structure outlined above, one can define quantities $\gamma=\left(1-\beta^{2}\right)^{-1 / 2}$ and $\theta$ by

$$
t_{ \pm}^{\mu}=\gamma\left(\beta V_{ \pm}^{\mu}+\cos \theta n_{ \pm}^{\mu}+\sin \theta z_{ \pm}^{\mu}\right)
$$

Here one has utilized the fact that the tangent vector is (for current purposes) a spacelike unit vector, and defined another spacelike unit vector $z$, that lies in the plane of the wormhole throat. Because the geodesic must, by construction, pass through the wormhole mouth in a smooth manner one must have the same coefficients $\gamma, \beta$, and $\theta$, occurring in each of these two equations. In particular, $t_{+} \cdot n_{+}=t_{-} \cdot n_{-}=\gamma \cos \theta$. One may now define the quantity $\ell_{ \pm} \equiv \gamma s_{ \pm}=s_{ \pm}\left(t_{ \pm} \cdot n_{ \pm}\right) / \cos \theta$. This represents the physical distance between the point $x$ and the relevant wormhole mouth, as measured in the rest frame of that wormhole mouth. By extension, one defines $\ell=\ell_{+}+\ell_{-}=s\left(t_{ \pm} \cdot n_{ \pm}\right) / \cos \theta$. To facilitate comparison with reference [9] one may define "time shifts" by $T_{ \pm} \equiv s_{ \pm}\left(t_{ \pm} \cdot V_{ \pm}\right)$. Further, one may define $T=T_{+}+T_{-}$. Finally, as a consistency check, observe $\delta\left(\eta_{+}\right)=\left(t_{+} \cdot n_{+}\right)^{-1} \delta(s)=$ $\left(t_{-} \cdot n_{-}\right)^{-1} \delta(s)=\delta\left(\eta_{-}\right)$. 
The van Vleck determinant is simply

$$
\Delta_{\gamma}(x, x)=\operatorname{det}\left(\delta^{\mu}{ }_{\nu}+\frac{s_{+} s_{-}}{s}\left[q^{\mu}{ }_{\nu}\right]\right)^{-1}
$$

As a result of all the preceding definitions and calculations, one extracts the relatively compact expression

$$
\begin{aligned}
{\left[q^{\mu}{ }_{\nu}\right] } & =\left[\kappa_{\mu \nu}(t \cdot n)+n_{\mu} n_{\nu}\left(\kappa_{\alpha \beta} t^{\alpha} t^{\beta}\right)(t \cdot n)^{-1}-\left\{\left(\kappa_{\mu \alpha} t^{\alpha}\right) n_{\nu}+\left(\kappa_{\nu \alpha} t^{\alpha}\right) n_{\mu}\right\}\right], \\
& =\gamma \cos \theta\left[\kappa_{\mu \nu}+n_{\mu} n_{\nu} \tan ^{2} \theta\left(\kappa_{\alpha \beta} z^{\alpha} z^{\beta}\right)-\tan \theta\left\{\left(\kappa_{\mu \alpha} z^{\alpha}\right) n_{\nu}+\left(\kappa_{\nu \alpha} z^{\alpha}\right) n_{\mu}\right\}\right] .
\end{aligned}
$$

Unfortunately this expression, while exact and general, is still too algebraically messy to be tractable.

\section{Radial impact}

Utilizing the machinery defined above one can define the notion of a geodesic that radially impacts on the mouth of the wormhole. A radially impacting geodesic is simply one for which $\theta=0$, so that one has

$$
t_{ \pm}^{\mu}=\gamma\left(\beta V_{ \pm}^{\mu}+n_{ \pm}^{\mu}\right)
$$

For instance: For spherically symmetric wormhole mouths that directly face one another, go to any Lorentz frame where the mouths have no transverse velocity, only longitudinal velocities. In any such frame pick any point on the line joining the two wormhole mouths. The geodesic from any such point to itself will impact the wormhole mouths radially in the sense described above. This is essentially the notion of the "central geodesic" as described by Kim and Thorne [7]. Returning to the general case, the virtue of radially impacting geodesics is that for such geodesics one has the great simplification $\kappa_{\mu \nu} t^{\mu}=0$, which implies

$$
Q_{\mu \nu} \equiv-R_{\mu \alpha \nu \beta} t^{\alpha} t^{\beta}=\delta(\eta)(t \cdot n)^{2} \kappa_{\mu \nu}=\delta(s)(t \cdot n) \kappa_{\mu \nu}
$$

Let me (temporarily) further restrict the analysis. For a spherically symmetric wormhole

of radius $R$, adopting the time shift identification of Kim and Thorne [7], the discontinuity in the second fundamental forms is: 


$$
\kappa_{\mu \nu}=\frac{2}{R} g_{\mu \nu}^{\perp}=\frac{2}{R}\left(g_{\mu \nu}+V_{\mu} V_{\nu}-n_{\mu} n_{\nu}\right)
$$

Inserting all of this machinery into the previously derived closed form expression for the single pass van Vleck determinant

$$
\begin{aligned}
\Delta_{\gamma}(s) & =\operatorname{det}\left(\delta^{\mu}{ }_{\nu}+\frac{s_{+} s_{-}}{s} \frac{2(t \cdot n)}{R}\left[g^{\perp}\right]_{\nu}^{\mu}\right)^{-1} \\
& =\left(1+\frac{s_{+} s_{-}}{s} \frac{2(t \cdot n)}{R}\right)^{-2}, \\
& =\left(1+\frac{\ell_{+} \ell_{-}}{\ell} \frac{2}{R}\right)^{-2} .
\end{aligned}
$$

This formula nicely interpolates between the near field and far field results of Kim and Thorne [7]. For points on the wormhole throat, compare this result to the $N=1$ case of Kim and Thorne's equation (48). For points far from the throat compare this result with the $N=1$ case of their equation (64). Emphasis should be placed on the fact that the relative velocity of the wormhole mouths is completely arbitrary. Likewise, the position of the point $x=y$, though constrained by the condition of radial impact is otherwise arbitrary.

A simple generalization is to note that there is now nothing sacred about a spherical wormhole mouth. As long as the geodesic is radially impacting one may write

$$
\begin{aligned}
\Delta_{\gamma}(x, x) & =\operatorname{det}\left(\delta^{\mu}{ }_{\nu}+\frac{s_{+} s_{-}}{s} \gamma \kappa^{\mu}{ }_{\nu}\right)^{-1} \\
& =\operatorname{det}\left(\delta^{\mu}{ }_{\nu}+\frac{\ell_{+} \ell_{-}}{\ell} \kappa^{\mu}{ }_{\nu}\right)^{-1} \\
& =\left(1+\frac{\ell_{+} \ell_{-}}{\ell} \frac{2}{R_{1}}\right)^{-1}\left(1+\frac{\ell_{+} \ell_{-}}{\ell} \frac{2}{R_{2}}\right)^{-1}
\end{aligned}
$$

Here, as previously, $R_{(1,2)}$ refer to the two principal radii of curvature, evaluated at the point where the geodesic impacts the wormhole mouth. Indeed, consider the cubical (or even polyhedral) wormholes of reference [5]. If the geodesic impacts radially on one of the flat faces, $R_{(1,2)}=\infty$, consequently $\Delta_{\gamma}(s)=1$.

If the base point $x$ is not on the throat of the wormhole then $\ell_{+} \ell_{-} \neq 0$. If the geodesic impacts radially on one of the edges, then one of the principal radii of curvature $R_{(1,2)}$ is zero, (the other is infinite). Consequently $\Delta_{\gamma}(s)=0$. 
If the base point $x$ is on the throat of the wormhole then $\ell_{+} \ell_{-}=0$. In this case, if the geodesic impacts radially on one of the edges, one is faced with making sense of the indeterminate form 0/0. Proceed as follows: The polyhedral wormholes of reference [5] were constructed by taking smooth cut-and-paste (thin throat) wormholes and considering the limit as one of the radii of curvature tends to zero. For the issue of interest, the indeterminate $0 / 0$ is resolved by observing that one should let the base point $x$ approach the wormhole mouth before letting the wormhole mouth acquire sharp edges by letting $R_{(1,2)} \rightarrow 0$. Consequently $\Delta_{\gamma}(s)=1$.

Another way of phrasing this is as follows: Letting the wormhole mouth acquire a sharp edge by letting $R_{2} \rightarrow 0$ is a somewhat dubious proposition once $R_{2}<\ell_{P}$. Radii of curvature smaller than the Planck length are of doubtful operational significance. Accepting that the struts supporting edges of a polyhedral wormhole have thickness of order the Planck length

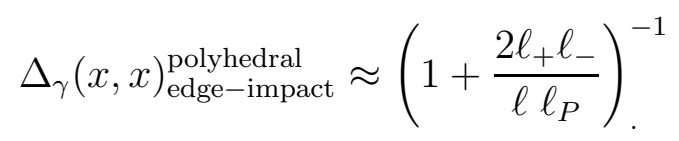

This "regulated" determinant is suitably well behaved as one approaches the throat.

\section{Generic impact}

To push the analysis beyond the case of radial impact is computationally messy. It is useful, in the interests of keeping the algebra from getting too unwieldy, to return to the case of spherical symmetry. Combining spherical symmetry with a generic impact angle

$$
\begin{aligned}
Q_{\mu \nu}(s) & =\delta(s)\left[\kappa_{\mu \nu}(t \cdot n)+n_{\mu} n_{\nu}\left(\kappa_{\alpha \beta} t^{\alpha} t^{\beta}\right)(t \cdot n)^{-1}-\left\{\left(\kappa_{\mu \alpha} t^{\alpha}\right) n_{\nu}+\left(\kappa_{\nu \alpha} t^{\alpha}\right) n_{\mu}\right\}\right] \\
& =\delta(s) \frac{2 \gamma \cos \theta}{R}\left[g_{\mu \nu}^{\perp}+n_{\mu} n_{\nu} \tan ^{2} \theta-\tan \theta\left\{z_{\mu} n_{\nu}+z_{\nu} n_{\mu}\right\}\right] .
\end{aligned}
$$

One is faced with the task of determining the eigenvalues of the matrix

$$
\left[\begin{array}{cccc}
0 & 0 & 0 & 0 \\
0 & \tan ^{2} \theta & -\tan \theta & 0 \\
0 & -\tan \theta & 1 & 0 \\
0 & 0 & 0 & 1
\end{array}\right]
$$


These eigenvalues are easily determined to be $\left(0 ; 0 ; 1 ; 1+\tan ^{2} \theta\right) \equiv\left(0 ; 0 ; 1 ; \sec ^{2} \theta\right)$. Consequently

$$
\Delta_{\gamma}(s)=\left(1+\frac{\ell_{+} \ell_{-}}{\ell} \frac{2}{R}\right)^{-1}\left(1+\frac{\ell_{+} \ell_{-}}{\ell} \frac{2 \sec ^{2} \theta}{R}\right)^{-1}
$$

Thus a radial impact is most effective at keeping the van Vleck determinant large. Grazing impacts lead to small values for the van Vleck determinant.

Note that as the base point of the geodesic approaches the mouth of the wormhole $\ell_{+} \ell_{-} \rightarrow$ 0 , so that (again) $\Delta_{\gamma}(x, x) \rightarrow 1$. The case $\theta=\pi / 2$, which corresponds to the geodesic having a tangential impact on the mouth of the wormhole, naively leads to the indeterminate form $0 / 0$. To regularize this, recognize that tangential impact $\theta=\pi / 2$, occurs only if one very carefully orients the two mouths of the wormhole to be facing away from each other. But it is impossible to hold the orientation of the wormhole mouths completely fixed. If nothing else, the Heisenberg uncertainty principle provides a fundamental limitation $\Delta \theta \Delta L \approx \hbar$ relating

the spread in orientation to the spread in angular momentum of the wormhole mouth. If one centers the orientation on $\theta=\pi / 2$, one has $\left\langle\sec ^{2} \theta\right\rangle \approx\left\langle\theta^{2}\right\rangle^{-1} \approx(\Delta L / \hbar)^{2} \equiv\left\langle J^{2}\right\rangle$. Thus a "regulated" van Vleck determinant, modified by orientational smearing may be taken to be

$$
\Delta_{\gamma}(x, x ; \theta=\pi / 2) \approx\left(1+\frac{\ell_{+} \ell_{-}}{\ell} \frac{2}{R}\right)^{-1}\left(1+\frac{\ell_{+} \ell_{-}}{\ell} \frac{2\left\langle J^{2}\right\rangle}{R}\right)^{-1}
$$

This "regulated" quantity tends to 1 as $x$ approaches the throat of the wormhole, which solves the problem of what to do with the indeterminate form.

\section{Double pass: exact result}

Consider a geodesic that starts at the point $x$, wraps through the wormhole twice, and returns to the point $x$. In view of the complexity of structure to be outlined below it is not particularly enlightening to even contemplate non radially impacting geodesics. Henceforth one specializes even further by assuming collinear motion for the wormhole mouths (i.e. 
radial approach or recession) and restricting attention to "central geodesics". The geodesic has three tangent vectors, $t_{ \pm}$, and $t_{0}$. One each of the two $t_{ \pm}$points towards each of the two wormhole mouths. $t_{0}$ points from one wormhole mouth to another. Each of the two wormhole mouths is characterized by a four-velocity $V_{ \pm}$. There are two normals to keep track of: $n_{ \pm}$. Here $n_{+}$is the normal to the wormhole mouth with velocity $V_{+}$, evaluated at the point where the geodesic from $x$ impacts that mouth. Similarly for $n_{-}$. One can define quantities $\gamma_{ \pm}=\left(1-\beta_{ \pm}^{2}\right)^{-1 / 2}$ by

$$
\begin{aligned}
& t_{ \pm}^{\mu}=\gamma_{ \pm}\left(\beta_{ \pm} V_{ \pm}^{\mu}+n_{ \pm}^{\mu}\right) . \\
& t_{0}^{\mu}= \pm \gamma_{ \pm}\left(\beta_{ \pm} V_{\mp}^{\mu}+n_{\mp}^{\mu}\right) .
\end{aligned}
$$

Here, as was the case for the $N=1$ case, one has utilized the fact that the tangent vectors are (for current purposes) spacelike unit vectors. Because the geodesic must, by construction, pass through the wormhole mouth in a smooth manner one must have the same coefficients $\gamma_{ \pm}$, and $\beta_{ \pm}$, occurring in these equations. In particular, $t_{ \pm} \cdot n_{ \pm}= \pm t_{0} \cdot n_{\mp}=\gamma_{ \pm}$. Because $V_{ \pm}$and $n_{ \pm}$are all coplanar,

$$
g_{\mu \nu}^{\perp}=\left(g_{\mu \nu}+V_{\mu}^{ \pm} V_{\nu}^{ \pm}-n_{\mu}^{ \pm} n_{\nu}^{ \pm}\right)
$$

This obviates the otherwise messy technical requirement of keeping track of precisely which of the $g^{\perp}$ one is dealing with, and permits the radical simplification

$$
\left[q_{\mu \nu}^{ \pm}\right]=\gamma_{ \pm} \frac{2}{R} g_{\mu \nu}^{\perp}
$$

So finally

$$
\Delta_{\gamma}(x, x)=\left(1+\frac{s_{+}\left(s_{-}+s_{0}\right)}{s} \frac{2 \gamma_{+}}{R}+\frac{s_{-}\left(s_{+}+s_{0}\right)}{s} \frac{2 \gamma_{-}}{R}+\frac{s_{+} s_{-} s_{0}}{s} \frac{4 \gamma_{+} \gamma_{-}}{R^{2}}\right)^{-2}
$$

This is the promised exact result for an arbitrary central geodesic that wraps twice through the wormhole.

One may still define the quantities $\ell_{ \pm} \equiv \gamma_{ \pm} s_{ \pm}=s_{ \pm}\left(t_{ \pm} \cdot n_{ \pm}\right)$, and interpret these quantities as the physical distance between the point $x$ and the relevant wormhole mouth, 
as measured in the rest frame of that wormhole mouth. The physical import of this result for the van Vleck determinant is unfortunately nowhere near as transparent as that derived for the case of a single pass.

\section{Multiple passes: exact result}

Most of the preparatory work for this section has already been done. For a completely general geodesic the algebraic complexity would be absolutely prohibitive. Accordingly, it is useful to specialize to the case of a completely smooth closed geodesic. One that wraps around the wormhole $N$ times.

Invoking the preceding multi-pass calculation, and now using the extended thin shell formalism to probe the Riemann tensor at the wormhole throat, one has

$$
s_{0}\left[q^{\mu}{ }_{\nu}\right]=s_{0} \gamma\left[\kappa_{\nu}^{\mu}\right]=\ell_{0}\left[\kappa_{\nu}^{\mu}\right]=\ell_{0}\left[\operatorname{diag}\left\{0,\left(2 / R_{1}\right),\left(2 / R_{2}\right), 0\right\}^{\mu}{ }_{\nu}\right] .
$$

The net result of the various factors of $\gamma$ has been to quietly disappear, after conveniently converting the arc-length along the geodesic into the physical distance between the wormhole mouths, as measured in the rest frame of the wormhole mouths.

Substitution into the previous results show

$$
\begin{aligned}
\Delta_{\gamma}^{-1}=\{1+ & \left.\frac{2 p_{+} p_{-} \ell_{0}}{R_{1}}\right\} \times\left\{1+\frac{2 p_{+} p_{-} \ell_{0}}{R_{2}}\right\} \\
& \times\left\{\sum_{n=0}^{N-1}\left(\begin{array}{l}
N+n \\
2 n+1
\end{array}\right)\left[\frac{2 \ell_{0}}{R_{1}}\right]^{n}\right\} \times\left\{\sum_{n=0}^{N-1}\left(\begin{array}{l}
N+n \\
2 n+1
\end{array}\right)\left[\frac{2 \ell_{0}}{R_{2}}\right]^{n}\right\} \times N^{-2}
\end{aligned}
$$

Equivalently

$$
\begin{aligned}
\Delta_{\gamma}=\{1+ & \left.\frac{2 \ell_{+} \ell_{-}}{\ell_{0} R_{1}}\right\}^{-1} \times\left\{1+\frac{2 \ell_{+} \ell_{-}}{\ell_{0} R_{2}}\right\}^{-1} \\
& \times N^{2} \times\left\{\sum_{n=0}^{N-1}\left(\begin{array}{l}
N+n \\
2 n+1
\end{array}\right)\left[\frac{2 \ell_{0}}{R_{1}}\right]^{n}\right\}^{-1} \times\left\{\sum_{n=0}^{N-1}\left(\begin{array}{l}
N+n \\
2 n+1
\end{array}\right)\left[\frac{2 \ell_{0}}{R_{2}}\right]^{n}\right\}^{-1} .
\end{aligned}
$$

Note that if $\ell_{0} \gg R_{(1,2)}$

$$
\begin{aligned}
\Delta_{\gamma} \approx\{1+ & \left.\frac{2 \ell_{+} \ell_{-}}{\ell_{0} R_{1}}\right\}^{-1} \times\left\{1+\frac{2 \ell_{+} \ell_{-}}{\ell_{0} R_{2}}\right\}^{-1} \\
& \times N^{2} \times\left\{\frac{4 \ell_{0}^{2}}{R_{1} R_{2}}\right\}^{N-1}
\end{aligned}
$$


Again, this result should be compared to eqs. (64) and (48) of Kim and Thorne [7]. The present result is compatible with, and at least along the central geodesic, a extension of the Kim-Thorne results.

\section{E. Discussion: isolated wormholes}

Having all these computations of the van Vleck determinant in isolated wormhole spacetimes in hand, I turn to the issue of Hawking's chronology protection conjecture. Recall that vacuum polarization effects disrupt the internal structure of the wormhole once any of the closed geodesics through the wormhole is shorter than

$$
\left.s\right|_{\text {disrupt }} \approx \max _{\gamma}\left\{\max _{x}\left\{\Delta_{\gamma}^{1 / 8}(x, x) \sqrt{\pi \ell_{P} R}\right\}\right\}
$$

The maximization runs over all closed geodesics $\gamma$ with base point $x$ on the throat of the wormhole.

In particular, let $\gamma_{1}$ be a geodesic that wraps only once through an isolated wormhole, then all the preceding calculations agree that when $x$ lies on the throat of the wormhole

$$
\Delta_{\gamma_{1}}(x, x)=1
$$

As the base point $x$ moves away from the throat of the wormhole, the van Vleck determinant may, and often will, rapidly fall off to zero. Nevertheless, for wormhole disruption effects, it is the behaviour at the throat that is relevant. One now has

$$
\left.s\right|_{\text {disrupt }} \approx \sqrt{\pi \ell_{P} R} \gg \ell_{P} .
$$

Which is what I wanted to prove.

\section{THE ROMAN CONFIGURATION}

The analysis of multiple wormhole configurations is a topic of considerable additional complexity. It is relatively easy to think up a putative time machine built out of two or more 
wormholes, each of which taken in isolation is not itself a time machine. The simplest such configuration was considered by Tom Roman [11, 33, 34], and uses two wormholes (vide [35]).

For example, for the time being ignore the internal structure of the two wormholes and simply model them by identifying two world-lines. Let wormhole \#1 be defined by

$$
(t, 0,0,0) \equiv\left(t+T_{1}, 0,0, \ell\right) .
$$

As long as the distance between the wormhole mouths $\ell$ is greater than the time shift $T_{1}$, wormhole \#1 does not, in and of itself, constitute a time machine. Now add a second wormhole. Let wormhole \#2 be offset a distance $\zeta$ along the $x$-axis. That is

$$
(t, \zeta, 0, \ell) \equiv\left(t+T_{2}, \zeta, 0,0\right)
$$

Again, as long as $\ell>T_{2}$, wormhole \#2 does not, in and of itself, describe a time machine. Now consider the null trajectory

$$
\begin{aligned}
(0,0,0,0) & \equiv\left(T_{1}, 0,0, \ell\right) \\
\rightarrow( & \left.T_{1}+\zeta, \zeta, 0, \ell\right) \equiv\left(T_{1}+\zeta+T_{2}, \zeta, 0,0\right) \\
& \rightarrow\left(T_{1}+\zeta+T_{2}+\zeta, 0,0,0\right) .
\end{aligned}
$$

This future-pointing null trajectory has returned to its spatial starting point in a total time $\Delta T=T_{1}+T_{2}+2 \zeta$. This total time shift can easily be arranged to be negative. (Example: $\ell=3 \zeta, T_{1}=T_{2}=-2 \zeta, \Delta T=-2 \zeta$.) We note that $\Delta T>-2 \ell+2 \zeta$, so that a necessary condition for this compound "Roman configuration" to form a time machine is $\ell>\zeta$. (The individual wormholes should permit jumps across the universe that are longer than the "offset" distance between wormhole \#1 and wormhole \#2.) The maximum possible size of the backward time jump is $2 \ell-2 \zeta$. One can add some internal structure to the wormhole by making the mouths spherically symmetric of radius $R$, as long as $R \ll \zeta$ this will not disturb the previous discussion. 


\section{A. van Vleck determinant: Roman configuration}

Evaluation of the van Vleck determinant for "Roman configuration" spacetimes is subtle. One shall soon see that it seems that vacuum polarization effects in such multiple wormhole putative time machines can, with a suitably bizarre choice of parameters, be arranged to be arbitrarily small at the onset of Planck scale physics. This is disturbing. (This property of Roman configuration wormhole systems has also been noted by Lyutikov [12], see the announcement in [1].)

Start the analysis by placing the wormhole mouths in general positions. All mouths are taken to be at rest with respect to one another, and wormhole \#1 is described by the identification

$$
\left(t, \vec{x}_{1}^{\text {in }}\right) \equiv\left(t+T_{1}, \vec{x}_{1}^{\text {out }}\right)
$$

Define $\ell_{1} \equiv\left\|\vec{x}_{1}^{\text {out }}-\vec{x}_{1}^{\text {in }}\right\|$, since one does not wish this wormhole to be a time machine in its own right $\left|T_{1}\right|<\ell_{1}$. For wormhole \#2 one simply copies all of these definitions, for example

$$
\left(t, \vec{x}_{2}^{\text {in }}\right) \equiv\left(t+T_{2}, \vec{x}_{2}^{\text {out }}\right)
$$

Now consider the following closed curve $C\left(t_{0}\right)$

$$
\left(0, \vec{x}_{1}^{\text {in }}\right) \equiv\left(T_{1}, \vec{x}_{1}^{\text {out }}\right) \rightarrow\left(t_{0}, \vec{x}_{2}^{\text {in }}\right) \equiv\left(t_{0}+T_{2}, \vec{x}_{2}^{\text {out }}\right) \rightarrow\left(0, \vec{x}_{1}^{\text {in }}\right)
$$

Here $t_{0}$ is a parameter that is for the time being arbitrary. We shall adjust $t_{0}$ in such a manner as to make $C$ a geodesic. The arc length along the curve $C\left(t_{0}\right)$ is

$$
s[C]=\sqrt{\left(\vec{x}_{1}^{\text {out }}-\vec{x}_{2}^{\text {in }}\right)^{2}-\left(T_{1}-t_{0}\right)^{2}}+\sqrt{\left(\vec{x}_{2}^{\text {out }}-\vec{x}_{1}^{\text {in }}\right)^{2}-\left(t_{0}+T_{2}\right)^{2}} .
$$

To simplify life, introduce the notation

$$
\begin{aligned}
& \ell_{1 \rightarrow 2} \equiv\left\|\vec{x}_{1}^{\text {out }}-\vec{x}_{2}^{\text {in }}\right\|, \\
& \ell_{2 \rightarrow 1} \equiv\left\|\vec{x}_{2}^{\text {out }}-\vec{x}_{1}^{\text {in }}\right\| .
\end{aligned}
$$

The arc length of the curve $C$ is extremized when 


$$
\frac{\partial s\left[C\left(t_{0}\right)\right]}{\partial t_{0}} \equiv \frac{T_{1}-t_{0}}{\sqrt{\left(\ell_{1 \rightarrow 2}\right)^{2}-\left(T_{1}-t_{0}\right)^{2}}}-\frac{T_{2}+t_{0}}{\sqrt{\left(\ell_{2 \rightarrow 1}\right)^{2}-\left(T_{2}+t_{0}\right)^{2}}}=0 .
$$

This equation has the parametric solution

$$
\begin{aligned}
& T_{1}-t_{0}=k \ell_{1 \rightarrow 2}, \\
& T_{2}+t_{0}=k \ell_{2 \rightarrow 1}, \\
& T_{1}+T_{2}=k\left(\ell_{1 \rightarrow 2}+\ell_{2 \rightarrow 1}\right) .
\end{aligned}
$$

So the arc-length of the closed geodesic of current interest, one that wraps once through the two wormhole system, obtained by setting $t_{0}$ to its critical value, is

$$
s[\gamma]=\sqrt{\left(\ell_{1 \rightarrow 2}+\ell_{2 \rightarrow 1}\right)^{2}-\left(T_{1}+T_{2}\right)^{2}}
$$

A putative time machine forms once $\left|T_{1}+T_{2}\right|>\ell_{1 \rightarrow 2}+\ell_{2 \rightarrow 1}$. This is a necessary and sufficient condition. But because one does not wish the individual wormholes to be time machines $\left|T_{1}\right|<\ell_{1},\left|T_{2}\right|<\ell_{2}$. Consequently $\left|T_{1}+T_{2}\right|<\left|T_{1}\right|+\left|T_{2}\right|<\ell_{1}+\ell_{2}$. So another necessary (but not sufficient) condition on the formation of a "two wormhole time machine" is $\ell_{1 \rightarrow 2}+\ell_{2 \rightarrow 1}<\ell_{1}+\ell_{2}$. This means that the net distance "jumped" through the two wormholes has to exceed the total distance travelled in going from one wormhole to the other.

One may also consider the behaviour of a future pointing null curve (not a closed curve) that threads the two wormholes

$$
\begin{aligned}
\left(0, \vec{x}_{1}^{\text {in }}\right) \equiv & \left(T_{1}, \vec{x}_{1}^{\text {out }}\right) \\
\rightarrow( & \left.T_{1}+\ell_{1 \rightarrow 2}, \vec{x}_{2}^{\text {in }}\right) \equiv\left(T_{1}+\ell_{1 \rightarrow 2}+T_{2}, \vec{x}_{2}^{\text {out }}\right) \\
& \rightarrow\left(T_{1}+\ell_{1 \rightarrow 2}+T_{2}+\ell_{2 \rightarrow 1}, \vec{x}_{1}^{\text {in }}\right)
\end{aligned}
$$

This curve returns to its spatial starting point in total time $\Delta T \equiv T_{1}+T_{2}+\ell_{1 \rightarrow 2}+\ell_{2 \rightarrow 1}$. As per the preceding discussion this can easily be negative. The virtue of this type of analysis is that it is now clear that the maximum possible size of the (single trip) backward time jump is $\Delta T_{\max } \equiv \ell_{1}+\ell_{2}-\ell_{1 \rightarrow 2}-\ell_{2 \rightarrow 1}$. 
Return to the general arguments given in the early portions of this paper. For a geodesic that makes a single pass through the mouths of two distinct wormholes $(N=2)$, one may still write down the closed form expression

$$
\begin{aligned}
A^{\mu}{ }_{\nu}(s)= & s \delta^{\mu}{ }_{\nu} \\
& +\Theta\left(s-s_{1}\right)\left\{\left(s-s_{1}\right)\left[q(1)^{\mu}{ }_{\nu}\right] s_{1}\right\} \\
& +\Theta\left(s-s_{2}\right)\left\{\left(s-s_{2}\right)\left[q(2)^{\mu}{ }_{\nu}\right] s_{2}\right\} \\
& +\Theta\left(s-s_{2}\right)\left\{\left(s-s_{2}\right)\left[q(2)^{\mu}{ }_{\rho}\right]\left(s_{2}-s_{1}\right)\left[q(1)^{\rho}{ }_{\nu}\right] s_{1}\right\} .
\end{aligned}
$$

Particularize to a closed geodesic, so that $x=y$. Let the base point $x$ lie on the mouth of wormhole \#1. Then $s_{1}=0$, and we may define $s_{1 \rightarrow 2}=s_{2}, s_{2 \rightarrow 1}=s-s_{2}$, so that $s=s_{1 \rightarrow 2}+s_{2 \rightarrow 1}$. Thus

$$
\Delta_{\gamma}(x, x)^{-1}=\operatorname{det}\left(\delta^{\mu}{ }_{\nu}+\frac{s_{1 \rightarrow 2} s_{2 \rightarrow 1}}{s}\left[q(2)^{\mu}{ }_{\nu}\right]\right)
$$

Now invoke the extended thin shell formalism to estimate $\left[q(2)^{\mu}{ }_{\nu}\right]$. For radial impact the previous arguments give $\left[q(2)^{\mu}{ }_{\nu}\right]=(t \cdot n)\left[\kappa(2)^{\mu}{ }_{\nu}\right]=\gamma\left(2 / R_{2}\right)\left[g^{\perp}(2)^{\mu}{ }_{\nu}\right]$. Here $\kappa(2)$ denotes the discontinuity in the second fundamental form at the throat of wormhole \#2. Assuming spherical symmetry I have taken $R_{2}$ to denote the radius of the throat. The factor $\gamma=(t \cdot n)$ is easily seen to be

$$
\gamma=\frac{\ell_{1 \rightarrow 2}}{s_{1 \rightarrow 2}}=\frac{\ell_{2 \rightarrow 1}}{s_{2 \rightarrow 1}}=\frac{\ell_{1 \rightarrow 2}+\ell_{2 \rightarrow 1}}{s_{1 \rightarrow 2}+s_{2 \rightarrow 1}}
$$

The net result is now

$$
\Delta_{\gamma}(x, x)=\left\{1+\frac{2 \ell_{1 \rightarrow 2} \ell_{2 \rightarrow 1}}{\left(\ell_{1 \rightarrow 2}+\ell_{2 \rightarrow 1}\right) R_{2}}\right\}^{-2} .
$$

This is compatible with the discussion on page 311 of [11], and for the case of a single trip through the compound system, generalizes that discussion to arbitrary placement of the wormhole mouths. See also [12]. 


\section{B. Discussion: multiple wormholes}

For a "Roman configuration" the wormhole disruption criterion may be given as

$$
\begin{aligned}
\left.s\right|_{\text {disrupt }} & \approx\left[R_{2}\left(\frac{1}{\ell_{1 \rightarrow 2}}+\frac{1}{\ell_{2 \rightarrow 1}}\right)\right]^{1 / 4} \sqrt{\pi \ell_{P} R_{1}} \\
& \approx \ell_{P}\left[\frac{R_{1}^{2} R_{2}}{\ell_{P}^{2}}\left(\frac{1}{\ell_{1 \rightarrow 2}}+\frac{1}{\ell_{2 \rightarrow 1}}\right)\right]^{1 / 4}
\end{aligned}
$$

The best possible case for the possibility of time travel is obtained if one makes $\Delta_{\gamma}(x, x)$ and hence $\left.s\right|_{\text {disrupt }}$ as small as possible, and pushes the disruption scale down into the Planck slop. This may be achieved by making $R$ as small as possible, and $\ell$ as large as possible. If one

tries to force $\left.s\right|_{\text {disrupt }}<\ell_{P}$ one acquires a constraint on the relevant wormhole parameters. Indeed

$$
R_{1}^{2} R_{2}<\ell_{P}^{2} \frac{\ell_{1 \rightarrow 2} \ell_{2 \rightarrow 1}}{\ell_{1 \rightarrow 2}+\ell_{2 \rightarrow 1}}<\ell_{P}^{2} \max \left(\ell_{1 \rightarrow 2}, \ell_{2 \rightarrow 1}\right)<\ell_{P}^{2} R_{\text {universe }} .
$$

Here the best possible case for time travel has been made by relaxing the separation of the wormholes as much as possible - surely the radius of the universe is a good upper bound on the distance between the wormholes. Take $R_{\text {universe }} \approx 3$ Giga-parsecs $\approx 6 \times 10^{60} \ell_{P}$. Then

$$
R_{1}^{2} R_{2}<6 \times\left(10^{20} \ell_{P}\right)^{3} \approx\left(3 \times 10^{-15} \mathrm{~m}\right)^{3} .
$$

So even with these ludicrously large separations between the wormhole mouths, one can only push the disruption scale down into the Planck slop by building the putative time machine out of even ludicrously smaller "traversable" wormholes with a radius of the order of femtometres. Since any would be time traveller would have to traverse the distance between wormhole \#1 and wormhole \#2 in normal space, he/she/it had better also be patient - a lifetime on the order of Giga-years would be appropriate.

If one attempts to build a "two wormhole time machine" on a more modest human scale one might take as a good bound $\max \left(\ell_{1 \rightarrow 2}, \ell_{2 \rightarrow 1}\right)<1 \mathrm{AU} \approx 9 \times 10^{45} \ell_{P}$. In this case

$$
R_{1}^{2} R_{2}<\left(2 \times 10^{15} \ell_{P}\right)^{3} \approx\left(10^{-20} m\right)^{3} \approx\left(\frac{\hbar}{20 \mathrm{TeV} / \mathrm{c}}\right)^{3} .
$$


So even if one lays hands on a couple of wormholes, and initiates suitable solar system scale engineering projects, any decent sized wormhole will be disrupted by vacuum polarization effects before time travel is achieved. If the wormholes in question are small enough one can push the disruption scale down below the Planck regime. This, of course does not mean one has proved that time travel is actually possible - all it means is that one has "fine-tuned" the system sufficiently to be in a parameter regime where one cannot trust even rudimentary calculations. Even if one were to then succeed in building a closed timelike loop, the small size of the wormhole would preclude anything short of a $20 \mathrm{TeV}$ quantum from getting through. This implies that one would need two SSC scale accelerators just to get a one-bit message through the putative time machine. Even if all of these constraints are satisfied, any single trip is limited to a maximum time jump of less than $(1 \mathrm{AU} / c) \approx 8$ minutes. This does not seem to be a useful workable recipe for studying tomorrow's Wall Street Journal.

\section{CONCLUSIONS}

The recent flurry of interest in traversable wormholes, with concomitant realization that it appears to be easy to turn a traversable wormhole into a time machine, has led to a great deal of interest. The issue of time travel is one that should be faced squarely as it cuts at the very foundations of what is currently believed about the structure of physics and the nature of the universe (multiverse?). While many authors are busy making their peace with the notion of time travel and its associated paradoxes, Hawking has promulgated his chronology protection conjecture whereby time travel is forbidden and the universe is made "safe for historians".

In an earlier paper [9] I argued that the universe appears to defend causality through a strategy of "defense in depth". This paper addresses the penultimate line of defense, vacuum polarization effects. This line of defense can only be formulated if one is in the semiclassical regime - a well defined background spacetime manifold is required, and one proceeds to perform quantum field theoretic calculations on that background. Vacuum polarization 
effects become infinite as one gets close to forming a time machine, thereby disrupting the traversable wormhole(s) used in the attempt to construct the time machine, and so aborting the formation of the time machine.

The technical aspects of this paper boil down to a minor agony of calculations of the van Vleck determinant in suitable model spacetimes that I believe mimic the essential features of traversable wormholes.

For putative time machines constructed out of a single wormhole, the evidence is conclusive that wormhole disruption occurs long before one enters the Planck regime.

For putative time machines constructed out of a two wormholes arranged in a "Roman configuration", the situation is messier. If the wormhole sizes and separations are human scale, then wormhole disruption occurs long before one enters the Planck regime. For suitably obtuse (not traversable by humans) choices of wormhole size and location one can push the disruption scale down below the Planck slop. Even begging the question of whether or not one can build or acquire traversable wormholes in the first place, this does not mean that a "Roman configuration" of wormholes can be used to build a time machine. All it means is that one's limited ability to calculate in the semiclassical regime has been completely obviated by entry into the uncharted wasteland of quantum gravity.

Perhaps the best attitude to take towards Planck slop effects on the chronology protection conjecture is that ultimately it should be taken to be an axiom, rather than attempting to prove it by calculation.

AXIOM: Quantum gravity, whatever it may be, is causal.

\section{ACKNOWLEDGMENTS}

I wish to thank Carl Bender, Kip Thorne, and Tom Roman for useful discussions. I also wish to thank Ian Redmount and Wai-Mo Suen for their helpful comments and the Aspen Center for Physics for its hospitality during an early phase of this work. This research was supported by the U.S. Department of Energy. 


\section{REFERENCES}

* $\quad$ Electronic mail: visser@kiwi.wustl.edu

[1] S. W. Hawking, in 6th Marcel Grossmann Meeting on General Relativity, Proceedings, Kyoto, Japan, 1991, edited by H. Sato, (World Scientific, Singapore, 1992).

[2] S. W. Hawking, Phys. Rev. D46, 603 (1992).

[3] M. S. Morris and K. S. Thorne, Am. J. Phys. 56, 395 (1988).

[4] M. S. Morris, K. S. Thorne, and U. Yurtsever, Phys. Rev. Lett. 61, 1446 (1988).

[5] M. Visser, Phys. Rev. D39, 3182 (1989).

[6] M. Visser, Nucl. Phys. B328, 203 (1989).

[7] S. W. Kim and K. S. Thorne, Phys. Rev. D43, 3929 (1991).

[8] G. Klinkhammer, Phys. Rev. D46, 3388 (1992).

[9] M. Visser, Phys. Rev. D47, 554 (1993).

[10] M. Visser, "Hawking's Chronology Protection Conjecture: singularity structure of the quantum stress-energy tensor," hepth@xxx/9303023, Nuclear Physics B (in press).

[11] K. Thorne, in GR13: General Relativity and Gravitation 1992, Proceedings of the 13'th international conference on general relativity and gravitation, Cordoba, Argentina, 1992, edited by R. J. Gleiser, C. N. Kozameh, and O. M. Moreschi, (I.O.P. Publishing, Bristol, 1993), pp. 295-315, \{see especially pp. 311-312\}.

[12] M. Lyutikov, "Vacuum polarization at the chronology horizon of the Roman spacetime", (1993), unpublished, result announced in [11.

[13] J. H. van Vleck, Proc. Natl. Acad. Sci. U.S.A. 14, 176 (1928).

[14] C. Morette, Phys. Rev. 81, 848 (1951). 
[15] W. Pauli, Pauli Lectures on Physics: Volume 6. Selected topics in Field Quantization, edited by: C. P. Enz, (MIT press, Cambridge, 1973), \{see especially pp. 161-174\}.

[16] B. S. De Witt and R. W. Brehme, Ann. Phys. (N.Y.) 9, 220 (1960) \{see especially pp. $226-235\}$.

[17] B. S. De Witt, in Relativity, Groups, and Topology, Proceedings of the Les Houches Summer School, Les Houches, France, 1963, edited by C. De Witt and B. S. De Witt, (Gordon and Breach, New York, 1964) \{see especially pp. 734-745\}.

[18] B. S. De Witt, Phys. Rep. 19, 295 (1975) \{see especially pp. 342-345\}.

[19] B. S. De Witt, in Relativity, Groups, and Topology II, Proceedings of the Les Houches Summer school, Les Houches, France, 1983, edited by B. S. De Witt and R. Stora, (North Holland, Amsterdam, 1984) \{see especially pp. 531-536\}.

[20] M. Visser, Phys. Rev. D47 (1993) 2395.

[21] C. W. Misner, K. S. Thorne, and J. A. Wheeler, Gravitation (Freeman, San Francisco, 1973).

[22] S. W. Hawking and G. F. R. Ellis, The large scale structure of space-time, (Cambridge University Press, Cambridge, 1973).

[23] I. S. Gradshteyn and I. M. Ryzhik, Table of integrals, series, and products, (Academic, New York, 1980).

[24] M. Abramowitz and I. A. Stegun, Handbook of mathematical functions, (Dover, New York, 1972).

[25] M. R. Spiegel, Mathematical handbook, (McGraw-Hill, New York, 1968).

[26] C. Lanczos, unpublished, (1922); Ann. Phys. (Leipzig) 74, 518 (1924).

[27] N. Sen, Ann. Phys. (Leipzig) 73, 365 (1924). 
[28] W. Israel, Nuovo Cimento 44B, 1 (1966); 48B, 463 (1967).

[29] A. Taub, J. Math. Phys. 21, 1423 (1970).

[30] C. Barrabes, Class. Quantum Gravit. 6, 581 (1989).

[31] I. H. Redmount, Mon. Not. R. Astr. Soc. 235, 1301 (1988).

[32] I. H. Redmount, Prog. Theoret. Phys. 73, 1401 (1985).

[33] T. Roman, private communication.

[34] K. S. Thorne, private communication.

[35] M. Visser, Phys. Rev. D 41, 1116 (1990) \{see especially p. 1121\}. 